\title{
Overview of current state of research on the application of artificial intelligence techniques for COVID-19
}

\author{
Vijay Kumar $^{1}$, Dilbag Singh ${ }^{2}$, Manjit Kaur ${ }^{2}$, Robertas Damaševičius ${ }^{\text {Corresp. 3,4 }}$ \\ ${ }^{1}$ CSED, National Institute of Technology, Hamirpur, Himachal Pradesh, India \\ 2 School of Engineering and Applied Sciences, Bennett University, Greater Noida, India \\ 3 Faculty of Applied Mathematics, Silesian University of Technology, Gliwice, Poland \\ 4 Department of Applied Informatics, Vytautas Magnus University, Kaunas, Lithuania \\ Corresponding Author: Robertas Damaševičius \\ Email address: robertas.damasevicius@vdu.It
}

Background Until now, there are still a limited number of resources available to predict and diagnose COVID-19 disease. The design of novel drug-drug interaction for COVID-19 patients is an open area of research. Also, the development of the COVID-19 rapid testing kits is still a challenging task. Methodology. This review focuses on two prime challenges caused by urgent needs to effectively address the challenges of the COVID-19 pandemic, i.e., the development of COVID-19 classification tools and drug discovery models for COVID-19 infected patients with the help of artificial intelligence (AI) based techniques such as machine learning and deep learning models. Results. In this paper, various Albased techniques are studied and evaluated by the means of applying these techniques for the prediction and diagnosis of COVID-19 disease. This study provides recommendations for future research and facilitates knowledge collection and formation on the application of the Al techniques for dealing with the COVID-19 epidemic and its consequences. Conclusions. The Al techniques can be an effective tool to tackle the epidemic caused by COVID-19. These may be utilized in four main fields such as prediction, diagnosis, drug design, and analyzing social implications for COVID-19 infected patients. 
1 Overview of current state of research on the application

2 of artificial intelligence techniques for COVID-19

3

4

5

6

7

8

9 10

11

12

13

14

15

16

17

18

19

20

21

22

23

24

25

26

27

28

29

30

31

32

33

34

35

36

37

38

39

Vijay Kumar ${ }^{1}$, Dilbag Singh², Manjit Kaur ${ }^{2}$ and Robertas Damasevicius ${ }^{3,4, *}$

\author{
${ }^{1}$ CSED, National Institute of Technology, Hamirpur, Himachal Pradesh, India \\ ${ }^{2}$ School of Engineering and Applied Sciences, Bennett University, Greater Noida, India \\ ${ }^{3}$ Faculty of Applied Mathematics, Silesian University of Technology, Gliwice, Poland \\ ${ }^{4}$ Department of Applied Informatics, Vytautas Magnus University, Kaunas, Lithuania
}

Corresponding Author: Robertas Damasevicius, Vileikos 8, Kaunas, Lithuania, Email address: robertas.damasevicius@vdu.lt

\title{
Abstract
}

Background Until now, there are still a limited number of resources available to predict and diagnose COVID-19 disease. The design of novel drug-drug interaction for COVID-19 patients is an open area of research. Also, the development of the COVID-19 rapid testing kits is still a challenging task. Methodology. This review focuses on two prime challenges caused by urgent needs to effectively address the challenges of the COVID-19 pandemic, i.e., the development of COVID-19 classification tools and drug discovery models for COVID-19 infected patients with the help of artificial intelligence (AI) based techniques such as machine learning and deep learning models. Results. In this paper, various AI-based techniques are studied and evaluated by the means of applying these techniques for the prediction and diagnosis of COVID-19 disease. This study provides recommendations for future research and facilitates knowledge collection and formation on the application of the AI techniques for dealing with the COVID-19 epidemic and its consequences. Conclusions. The AI techniques can be an effective tool to tackle the epidemic caused by COVID-19. These may be utilized in four main fields such as prediction, diagnosis, drug design, and analyzing social implications for COVID-19 infected patients.

\section{Introduction}

The novel coronavirus has been reported in Wuhan (China) in December 2019. Wuhan became the epicenter of coronavirus [1]. Coronavirus infected 138,987,378 persons and 2,988,860 deaths in 210 countries as on 15 April, 2021 [2,3]. World Health Organization (WHO) entitled the disease caused by coronavirus as COVID-19 and declared it an epidemic in February 2020 [4]. The virus, also known as SARS-CoV-2, is a novel and evolving virus. The treatment of SARS-CoV-2 is based on the symptoms present in the patient. The most common and specific symptoms are fever and cough with some other non-specific symptoms such as fatigue, headache, and dyspnea [5]. Supplement Table 1 shows the contribution of these symptoms in infected persons [6]. The common transmission methods are human contact and respiratory droplets. The COVID infection 
40 depends upon age, preexisting health conditions, hygiene and social habits, location, and frequency

41 of person interactions [7].

43 Supplement Table 2 shows the estimation of the severity of COVID-19 disease infected peoples $44[8,9]$. The risk associated with infection is broadly classified into three categories namely infection 45 risk, severity risk, and outcome risk [10]. The infection risk is associated with a specific 46 group/person having COVID-19. The person/group having severe symptoms of COVID-19 and 47 require intensive care and hospitalization is known as severe risk. If the treatment is not effective towards the infected person/group then there is less possibility to recover or die. Self-isolation and social distancing are the most effective strategy to alleviate this epidemic. Isolation and house quarantine are core strategies to alleviate the infectious disease and reduce the transmission via diminishing the contact of those that are infected [11]. Most governments have imposed lockdown to save lives. However, the economy of every country was greatly affected by the lockdown process. The Organization of Economic Cooperation and Development declared that the growth rate may be slow down by $2.4 \%$ [12].

The main problems associated with coronavirus's pandemic can be resolved through Artificial Intelligence (AI) [13]. AI has the potential to screen the population and predicting the risk of

58

59

60

61

62

63

64

65

66

67

68

69

70

71

72

73

74

75

76

77

78

79 infection. The prediction process utilizes information such as how much time a person spends in a highly infected area and how many persons are infected in that region. AI developed a spatial prediction model based on this information and envisage the infection transmission [14]. AI-based prediction model uses information about the infected person through the symptoms. Coronavirus infected person does not show the symptoms in most of the cases. Due to this, it is very difficult to detect an infected person. The same thing has been reported in Wuhan city. In Wuhan, $50 \%$ of the infected persons are asymptomatic carriers [15]. The exhaustive testing of coronavirus is required to develop a better predictive model. The same scenario has been implemented in South Korea to prevent the spread of virus infection. The exhaustive testing produces a large amount of data about the infected and non-infected person. Based on these data, AI can be used to suppress the spread of infection, development of vaccines, diagnosis, and social-economic impact $[16,17]$. Recently, most of the AI researchers are working on the above-mentioned areas. The number of preprints available on the Internet is a witness of this work [18]. Recently, many medical image processing techniques based upon chest $\mathrm{CT}$ and chest X-ray images have been considered. Also, meta-heuristics techniques can be useful to diagnose COVID-19 patients [19].

By the end of November 2020, more than 75,000 scholarly articles were published and indexed on Pubmed on COVID-19 [20]. However, these articles did not address in-depth the key issues in applying computational intelligence to combating the COVID-19 pandemic. Thus, it is time to discuss and summarize studies related to artificial intelligence from such a large number of articles. Considering the above observations, now it is the time to systematically categorize and review the current progress of research on artificial intelligence. Accordingly, this survey aims to assemble 
80 and summarize the highlights of the latest developments and insights in applying artificial

81 intelligence approaches, such as machine learning, and deep learning, to practical applications used

82 to fight against COVID-19.

83

84 The principal contributions of this review paper are as follows:

851 . The impact of artificial intelligence techniques is assessed on early cautionary and vigilant 86 systems.

87 2. COVID-19 datasets and visualization techniques are discussed with their applications.

88 3. AI-based diagnosis and treatment of COVID patients are assessed.

89 4. The impact of AI on drug discovery and design is evaluated.

90 5. The effect of COVID-19 is evaluated on social and economic aspects.

91

92

93

94

95

96

97

98

99

100

101

102

103

104

105

106

107

108

109

110

111

112

113

The remaining paper is organized as follows. Section 2 presents a description of our methodology used. Section 3 presents a short description of coronavirus. The data gathering tools and techniques are discussed in Section 4. Section 5 presents the prediction models. The diagnosis of infected patients is presented in Section 6. Section 7 describes the treatments of COVID patients. The drug discovery and design of vaccines are presented in Section 8 . The social and economic impacts are discussed in Section 9. Future research directions are discussed in Section 10. The concluding remarks are drawn in Section 11.

\section{Research Methodology}

In this section, the surveys related to application of artificial intelligence towards COVID-19 are discussed followed by the research paper selection methodology used in this paper.

\section{a. Related surveys}

Since the start of the COVID-19 pandemic, many research paper was published (just Scopus has already indexed over 50000 papers alone). Consequently, several surveys and systematic review studies have tried to systemize and summarize the state of research and knowledge in this emerging research sub-field, including on the use of artificial intelligence methods. Related survey papers are summarized in Supplement Table 3 and discussed in more detail below.

Albahri et al. [21] reviewed the state-of-the-art techniques for coronavirus prediction algorithms based on data mining and ML assessment. They used Preferred Reporting Items for Systematic Reviews and Meta-Analyses (PRISMA) as a methodological guideline. The main focus of the survey study was on the development of different AI and ML applications, systems, algorithms, methods and techniques. However, only eight articles were fully evaluated and included in this review, which outlined the insufficiency of research in this important area.

118

119

Lalmuanawma et al. [22] aimed to review the role of AI and ML as one significant method in the arena of screening, predicting, forecasting, contact tracing, and drug development for SARS-CoV- 
120

121

122

123

124

125

126

127

128

129

130

131

132

133

134

135

136

137

138

139

140

141

142

143

144

145

146

147

148

149

150

151

152

153

154

155

156

157

158

159

2. The study concluded that the use of modern technology with AI and ML dramatically improves the screening, prediction, contact tracing, forecasting, and drug/vaccine development although they also noted the lack of deployment of AI models to show their real-world operation.

Ozsahin et al. [23] analyzed the use artificial intelligence (AI) techniques to diagnose COVID-19 with chest computed tomography (CT). Their study included 30 articles from ArXiv, MedRxiv, and Google Scholar identified using the selective assessment method.

Pham et al. [24] present an overview of AI and big data, then identify the applications aimed at fighting against COVID-19, next highlight challenges and issues associated with state-of-the-art solutions, and finally come up with recommendations for the communications to effectively control the COVID-19 situation. They based their study on the selected assessment of peerreviewed papers and preprints from IEEE Xplore, Nature, ScienceDirect, Wiley, arXiv, medRxiv, and bioRxiv.

Rasheed et al. [25] presented the collation of the current state-of-the-art technological approaches applied to the context of COVID-19, while covering multiple disciplines and research perspectives.

Tseng et al. [26] focused on categorizing and reviewing the current progress of computational intelligence for fighting COVID-19, which additionally to machine learning and neural networks also discuss fuzzy logic, probabilistic and evolutionary computation based methods. Tayarani [27] presented the applications of artificial intelligence techniques in COVID-19. They discussed the machine learning techniques for prediction and treatment of infected persons.

However, since the body of knowledge on COVID-19 related research problems is rapidly updated and supplemented, there is a need to provide a new survey of papers to summarize the most recent state-of-the-art in the application of AI techniques for in the COVID-19 related research fields.

\section{b. Survey Methodology}

This survey capitalizes on previous literature to describe approaches for handling COVID-19 that can empower the research community to develop new AI-based methods for the prediction of cases, diagnosis of patients, drug discovery, and design of vaccines.

Similarly to the survey of Lalmuanawma et al. [22] and Pham et al. [24] we used the selective assessment method, while similarly to the survey of Ozsahin et al. [23] we focused on less formal databases such as BiorXiv and ArXiv, which allows to analyzes the most recent trends in research without waiting for formal publication in indexation by major databases, which can take a significant amount of time. Due to the nature of this study being a relatively new research subject, we mostly focused on the pre-print papers.

The rationale for this survey is the extreme growth of research papers published on COVID-19 papers, which requires them to be analyzes, categorized, and reflected upon. Fig. 1 shows the 
160

161

162

163

164

165

166

167

168

169

170

171

172

173

174

175

176

177

178

179

180

181

182

183

184

185

186

187

188

189

190

191

192

193

194

195

196

197

198

199

number of research papers posted on BiorXiv, ArXiv, and MedRxiv from January, 2020 to April 2021. This study is intended for researchers working in the intersection of computer science, artificial intelligence, and biomedical domains.

This survey covers literature published in 2020 (January-September) in different fields of study related to COVID-19. Because no similar efforts have yet been made, we draw on examples from the previous literature. Unbiased and comprehensive coverage of the previous literature was accomplished by querying online preprint research archives (BiorXiv, ArXiv, and MedrXiv) with search terms directly relates to COVID-19 (e.g., "covid-19", "coronavirus", "SARS-CoV-2") and with Boolean operators to find studies applying artificial intelligence (e.g., "artificial intelligence" AND "neural networks"). Studies were included if they provided an overview of open science concepts that were relevant to addressing COVID-19. Once the papers were selected, they were categorized and analyzed based on their research aims and approaches used. Supplement Table 4 shows the selection and exclusion criteria for shortlisted research articles.

\section{Coronavirus Overview}

A coronavirus is a group of viruses that can be transferred between human beings and animals. The novel coronavirus is known as SARS-CoV-2. Coronavirus $(\mathrm{CoV})$ belongs to the family Coronavirinae of the order Nidovirales [29]. $\mathrm{CoV}$ is broadly classified into four main classes namely $\alpha, \beta, \gamma$, and $\delta$. The first two (i.e., $\alpha$ and $\beta$ ) contaminate mammals only. The later ones (i.e., $\gamma$ and $\delta$ ) contaminate birds. They may contaminate mammals in a rare case. The genome of $\mathrm{CoV}$ consists of a single-stranded positive-sense RNA whose length is $30 \mathrm{~kb}[30]$. CoV is the largest among the existing RNA viruses. It has also a 5' cap and 3' poly-A tail [30].

Based on the literature available, CoVs infecting human beings include two $\alpha$-CoVs (229E and NL63), and five $\beta$-CoVs (OC43, HKU1, MERS-CoV, SARS-CoV, and SARS-CoV-2. SARS-CoV-2 is a novel class of $\beta$-coronavirus genera that consists of bat-SARS-like (SL)-CoV ZC45, bat-SL-CoV ZXC21, SARS-CoV, and MERS-CoV. From recent studies, it found that SARS-CoV-2 came from wild animals. However, the exact source of this virus is unknown. There is a substantial genetic difference between now and SARS-CoV and numerous similarities among them in terms of chemical and physical characteristics [31].

\section{Data Gathering Systems for COVID-19}

As we knew that the COVID-19 is an infectious disease. The spread of this virus can be stopped through no human interaction. AI-based tools are used to collect the COVID-19 data, develop a vigilant system, and visualize the COVID-19 data [32]. Recently, smartphone applications are also developed to diagnose the user's health and trace the spread of infection. The main intention behind these applications is to identify vulnerable communities, provide real-time information to both 
200 patients and medical staff, detect infected hotspot areas, and generate advice for patient's health 201 [33]. Fig. 2 shows the categorization of data gathering systems for COVID-19.

202

203

204

205

206

207

208

209

210

211

212

213

214

215

216

217

218

219

220

221

222

223

224

225

226

227

228

229

230

231

232

233

234

235

236

237

238

239

\section{a. An early cautionary and vigilant system}

BlueDot is an effective analysis tool, which is developed in Canada [34]. It utilizes natural language processing (NLP) and machine learning technique. It was able to identify the outbreak of COVID-19 and generated vigilant alerts to users. It was used to generate the cautionary warnings to cities where the people reached from Wuhan city after January 2020 [35]. In Belgium, the telecom operators are integrated with healthcare for analysing the infection spread in particular areas and identifying the infected hotspot areas. They have segregated the population into different regions according to the spread of infection. The same concept has also been used in some other countries. It provides real-time monitoring of patients and the consultant can use this information to prepare the prevention plans for virus infection in time [36].

Similarly, Austria Telecom has agreed with its authorities to deliver the customer data. The customer data is used to trace their movements in the hotspot Lombardy region. MIT's consortium is working on a smartphone application to detect the spread of virus infection. Global Positioning System (GPS) in a smartphone is used for checking the intersection of user's trails with trails of infected persons. They used cryptographic techniques to protect the data. The application generates early cautionary signs to analyse the risk of infection spread after contacting with the infected persons. Instagram developed a COVID-19 tracker named as 'RT.live'. It shows the state-by-state information on coronavirus infection in the US. The data analysis algorithm is used to estimate the reproduction of virus infection.

'HealthyTogether' application is developed by the Utah government for reducing the spreading of COVID-19. It is used to analyze the symptoms, determine the nearby testing center, and assessment of test results. Singapore Government agency developed a 'TraceTogether' application to protect the community from COVID-19. It helps contact tracers notify quickly through Bluetooth connection.

India Government developed the 'Aarogya Setu' application to monitor the infection caused by COVID-19 patients. This application has used a questionnaire to determine whether the person is infected or not. It is helpful to find if the infected patient was somewhere near the user. Another vigilant system was HealthMap to impose quarantines and restrict the movement of peoples.

Supplement Table 5 shows a brief description of the vigilant system for COVID-19. Supplement Table 6 depicts the functionality analysis of different real-time surveillance mobile apps for COVID-19 [37].

\section{b. Data visualization systems}


240

241

242

243

244

245

246

247

248

249

250

251

252

253

254

255

256

257

258

259

260

261

262

263

264

265

266

267

268

269

270

271

272

273

274

275

276

277

278

279

Data visualization techniques/dashboards utilized AI for tracking and forecasting of COVID infection. They provide a global overview of infected persons. Data visualization system is categorized into two broad categories namely local and global dashboards [38]. The Center for System Science and Engineering of Johns Hopkins University developed the COVID-19 dashboard (JHU-CSSE) for global viewing of COVID-19. NextStrain generates the genomic epidemiology of COVID-19. It consists of a dropdown list for Asia, Africa, Europe, and Oceania. BBC dashboard provides the visualization of coronavirus infected areas in the World. It provides the effect of lockdown on the death cases in six countries namely the UK, Italy, Spain, France, Germany, and the US. The New York Times offers the dashboard for virus infection in various areas of the World [39]. HealthMap uses the World map for showing the visualization of infected cases in a particular area. Bing's AI tracker is used to show infected, recovered, and fatal cases of different parts of the World. The filter is applied to a particular country to show the infected persons in a particular province. South Africa developed COVID-19 ZA South Africa dashboard for tracking the infected, recovered, and death cases in South Africa. It also tracks the number of tests conducted and positive cases in different provinces. Tableau implemented a COVID-19 Data Hub for a daily global tracker. COVID19 India is initiated by crowdsourcing. The infected, recovered, and deaths are shown in both tabular and graphical manner. Supplement Table 7 depicts the comparative analysis of different dashboards concerning the statistical information reported.

\section{COVID-19 datasets and resources}

Artificial Intelligence techniques require big data for further assessing the drug discovery, risk assessment, spread of infection, treatment, and cure of COVID infected patients. The datasets are broadly classified into text, social media, biomedical, speech, and case studies. The classification of COVID-19 datasets is shown in Fig. 3.

\section{i. Text Datasets}

The text datasets include risk factors, non-pharmaceutical interventions, incubation period, the spread of virus infection, and stability of the environment [40, 41]. WHO Global Research Database provides scientific information on COVID-19. COVID-19 Open Research Dataset is an open dataset. The Kaggle challenges provide the COVID-19 data for analysis. LitCOVID and AI COVID-19 dataset consist of clinical trial data.

\section{ii. Social Media Datasets}

COVID-19 Tweets and Covid-19 Twitter dataset comprise of coronavirus related tweets, which are misclassified information and rumors on Twitter [42]. These datasets contain the reactions from different persons on the tweets [43]. COVID-19 Real World Worry Dataset [44] consists of labeled texts of persons' emotional responses towards COVID-19. It also consists of public 
280

281

282

283

284

285

286

287

288

289

290

291

292

293

294

295

296

297

298

299

300

301

302

303

304

305

306

307

308

309

310

311

312

313

314

315

316

317

318

319

sentiment and the mental health of persons in the infected areas of the World. Institutional, News, and Media Tweet Dataset consists of institutional communication of COVID infected areas in different countries. Both COVID-19 Coronavirus News Article dataset and COVID-19 Television Coverage dataset comprises of covering the news and prints of COVID-19 outbreak. These datasets cover the spreading of the virus, misinformation about drugs, and tracking of coronavirus.

\section{iii. Biomedical Datasets}

Covid Chest X-ray dataset is available as an open-source dataset. It consists of medical images that can be used to train deep learning/machine, learning models [45]. Data labeling and annotation can be used by radiologists. COVID-19 Survival Calculator uses the symptoms of infected patients. However, these are unmanageable and require manual maintenance. There are several data repositories, which provide links to researchers to easily access the data. Data4COVID Living Data and COVID-19 Dataset Clearinghouse repositories have many links to coronavirus data from different parts of the world. AI-based tools and techniques are used to extract the data from repositories, cleaning the data, and develop some predictions on cleaned data. The gene structure of SARS-CoV-2 should be known for the development of drugs and antibodies. Nextstrain is working on the gene structure of SARS-CoV-2 to illustrate the spread of coronavirus. RCSB Protein Data Bank and Global Health Drug Discovery Institute developed online COVID data portals for analysis of protein structure. American Chemical Society issued an open Covid-19 antiviral candidate compounds dataset that may be useful for the treatment of coronavirus infected patients. The crowdsourced developed a Fold.it [46] science game to design antiviral protein for fighting against COVID-19.

\section{iv. Speech Datasets}

Speech dataset consists of either cough sounds or breathing rate, which are used to detect the COVID-19. The smartphone and telemedicine can be used to collect the samples of cough and breathe of patients. Some researchers designed a cross platform for collection of both cough and breath). COVID-19 can be differentiated through the voice sounds. The scholars from the University of Cambridge made calls for voice sample collection from the participants. They made open source repository for cough samples.

\section{v. Case Studies}

WHO and the National Centers for Disease Control released the COVID datasets for tracking the progress of infection and perceiving the effect of preventive measures. Johns Hopkins and Github [47] offer information about COVID-19 infected areas. Humanitarian Data Exchange hosted data about the coronavirus. CHIME is a COVID-19 Hospital Impact Model for Epidemics based SIR modeling that utilizes the number of infected, recovered, and fatality persons to predict the 
320

321

322

323

324

325

326

327

328

329

330

331

332

333

334

335

336

337

338

339

340

341

342

343

344

345

346

347

348

349

350

351

352

353

354

355

356

357

358

359

infection caused by coronavirus and the requirement of hospital beds. Supplement Table 8 depicts the downloadable links and description of datasets [48].

\section{Prediction Models}

AI-based prediction models can be used to predict the mortality rate, infection in patients through cough and biomarkers, genome structure associated with fatality rate, and severity rate. The epidemic of the tracker was developed by Metabiota. It utilized the forecasting machine learning model used for the prediction of infection spread. Robert Koch Institute developed a SIR prediction model, which uses quarantines, social distancing, and lockdowns. This model was implemented in the $\mathrm{R}$ language. It was helpful to reduce the spreading of infections.

Yan et al. [49] proposed a machine learning technique to predict the severity of COVID-19. The survival rate of patients at Tongji Hospital in Wuhan is assessed through the prognostic biomarker. The prediction accuracy obtained from the proposed machine learning technique was approximately $90 \%$. Jiang et al. [50] developed an AI framework that has the predictive capability to analyze the severity of patients. They developed an algorithm to identify the clinical characteristics of infected persons. The predictive model used clinical characteristics to predict the severe illness of fifty-three patients with $80 \%$ accuracy. The predictive model is tested on two hospitals in Wenzhou, Zhejiang, China.

Alotaibi et al. [51] used machine learning techniques namely Support Vector Machine (SVM), Artificial Neural Network (ANN) and Random forest to predict the severity of infected patients. The prediction accuracies obtained from SVM, ANN, and Random forest are $86.67 \%, 83.33 \%$ and $90.83 \%$, respectively. Chatterjee et al. [52] developed a susceptible, exposure, infectious and recovered (SEIR) model to study the impact of healthcare during the pandemic situation. By using this model, hospitalizations and ICU requirements can be reduced to $90 \%$. Ghosal et al. [53] used a linear regression to predict the number of deaths in India. The predicted death rate is 211 and 467 by the end of $5^{\text {th }}$ and $6^{\text {th }}$ week, respectively.

Imarn et al. [54] developed the AI4COVID-19 tool for the preliminary diagnosis of COVID-19. AI4COVID used a two-second cough recording of infected persons. After the analysis of cough samples, the AI tool generates preliminary diagnosis for patients. AI4COVID distinguished COVID and non-COVID patients with 90\% accuracy. Feng et al. [55] developed a diagnosis model for the preliminary identification of infected patients. The prediction was based on travel history, clinical symptoms, and test results. Lasso regression applied to features obtained from clinical symptoms for prediction. The features are extracted from Rao et al. [56] implemented machine learning algorithms to determine the possible causes of coronavirus in the quarantine areas through a mobile-based survey. The algorithms can easily predict the no-risk, moderate risk, and high risk of infection through travel history and contact with infected persons.

Peer) Comput. Sci. reviewing PDF | (CS-2020:09:52767:1:2:NEW 27 Apr 2021) 
360

361

362

363

364

365

366

367

368

369

370

371

372

373

374

375

376

377

378

379

380

381

382

383

384

385

386

387

388

389

390

391

392

393

394

395

396

397

398

399

Tang et al. [57] implemented a machine learning technique for automatic severity assessment of infected patients using chest $\mathrm{CT}$ images. They extracted features from lungs and applied them to a random forest model for predicting the severity of COVID-19. The accuracy obtained from the random forest model was $87.50 \%$. Qi et al. [58] used logistic regression (LR) and random forest (RF) to extract the features from 72 pneumonia lesions of 31 patients. The sensitivity and specificity obtained from LR are 1.0 and 0.89 , respectively. However, the RF model provided a sensitivity of 0.75 and a specificity of 1.0. Patrikar et al. [59] modified the SEIR framework to study the effect of social distancing on the spread of coronavirus. They found that the infection can be reduced to $78 \%$ through social distancing.

Supplement Table 9 shows the comparative analysis of prediction models for COVID-19.

\section{Screening Techniques for COVID-19}

The diagnosis of a COVID-19 patient is a challenging task. The testing of every patient is timeconsuming. Due to the pandemic situation, faster and cheaper tests are required to generate the medical report. AI-based techniques are used to screen COVID-19 patients. There are three different methods to screen patients using AI. These are face recognition, wearable devices, and virtual healthcare assistant (see Fig. 4) [60].

\section{a. Screening through patients' face}

Face recognition technology is used to eliminate the spread of infection caused by coronavirus through human contact. It can be used with a temperature detection tool for the efficient identification of COVID-19 patients. Drones/Robots equipped with the thermal scanner are used for detecting fever in patients from an appropriate distance. Chinese firm Baidu developed infrared cameras to scan the crowds for temperature scanning [61]. They can screen hundreds of persons within one minute. However, the fever can be wrongly detected if a person wears some items on the face. AI-based face recognition tools can be installed at schools, colleges, railway, airport, and community places. These tools can automatically detect the persons having fever, tracing their positions, and detect whether the person has a mask or not.

\section{b. Screening through a wearable device}

Nowadays, wearable health devices such as Fitbits and Garmins are more popular to monitor physiological parameters such as heart rate, blood pressure, oxygen levels, body temperature, movement, and sleep for a better lifestyle. Apple developed an AI-based watch to determine the temperature and heart rate to identify the symptoms of patients [62]. OURA developed an activity tracking ring that uses body temperature, breathing rate, and heart rate to determine the onset patterns, progress, and recovery of the patient [63]. Stanford Medicine and Google company came 
400 together to utilize the data collected from the wearable device to detect the symptoms of an infected 401 person. They used body temperature and heart rate for fighting against the COVID-19 infection. 402 Central Queensland University collaborated with Cleveland Clinic to analyze the data collected 403 from Whoop's wearable devices [64]. Shanghai Public Health Center has developed in-built 404 temperature sensors in wearable devices to detect the body temperature for COVID-19 patients 405 regularly. The detected temperature is continuously sent to the nursing station for patient 406 monitoring. Canada based Proxxi technologies developed a wrist device named 'Halo' that 407 communicate to user through vibration [65]. It gives an alert to the user about his come within 408 range of 6 feet of another wearable user. It uses Bluetooth technology to communicate with others 409 and keep the data record about users whom the wearable user met.

410

411

412

\section{c. Screening through Chatbots}

In this pandemic situation, chatbot developers and healthcare systems are integrated for the prediction of infected patients. Chatbots are used as an accelerator tool for the healthcare of COVID infected persons. AI-based Stallion used the capabilities of natural language processing (NLP) to develop a Chatbot as a virtual healthcare agent [66]. It endorses protection measures, monitors symptoms, and generates suggestions to individuals for home quarantine or hospital admissions.

Some countries developed a "Self-Triage" system that uses a questionnaire about the symptoms of patients. Microsoft developed a healthcare virtual assistant that will help to determine the appropriate action using the symptoms of patients. They included risk assessment, clinical triage, and COVID-19 question-answering in their chatbot [67].

Google Cloud developed a virtual assistant that will provide information about COVID-19. The virtual assistant quickly responds to questions raised by users and provide optimal information to users [68]. BITS students developed a chatbot to yield awareness among the users. AI-enabled doctor video bot, named AskDoc, is developed to provide answers about the COVID-19 queries using voice and text. Facebook implemented a WhatsApp bot to support the users to update them on the outbreak of COVID-19 [69]. Zoe chatbot gives users answers and appropriate information [70].

Supplement Table 10 shows the AI-based tools/techniques for the screening of COVID-19 symptoms.

\section{Diagnosis of COVID-19}

In this pandemic situation, a quicker diagnosis is required. The most widely used technique for diagnosis is a real-time reverse transcription-polymerase chain reaction (RT-PCR) [71]. The well- 
439 known radiological imaging techniques are X-ray and computed (CT) [72]. Due to less sensitivity $440(60 \%-70 \%)$ of RT-PCR, symptoms can be detected through radiological images [73]. However, 441 radiological images are sensitive to detect the infection caused by COVID-19 and can be used to 442 monitor the patients [74]. A less number of clinical expertise is available as compared to the 443 COVID-19 cases that arose in this pandemic situation [75]. Therefore, AI-based tools and 444 techniques can be used for faster diagnosis.

445

446

447

448

449

450

451

452

453

454

455

456

457

458

459

460

461

462

463

464

465

466

467

468

469

470

471

472

473

474

475

476

477

478

\section{a. Radiological Imaging}

Researchers studied 33\% of chest CTs have rounded lung opacities. It is observed from the CT scan that symptoms may not be detected in the initial two days [76]. The abnormal finding is detected in the CT scan of patients after ten days of symptoms observed [77]. Initially, due to the low sensitivity of RT-PCR kits, clinical experts suggested using Chest CT for diagnosis [78]. The traditional methods take 15 minutes for analysis of the chest CT scan. Nowadays, machine learning or deep learning techniques are used for automated analysis of CT scans and chest X-rays. These techniques will help to speed up the analysis process [79, 80, 81]. Fig. 5 depicts the distribution of radiological images for diagnosis of COVID-19.

\section{i. Al Tools for Radiological Images}

Baidu's team developed a LinerFold software that diagnoses the infection of COVID-19 in 27 seconds [82]. The prediction time is reduced from 55 minutes to 27 seconds and helps in developing the drug for coronavirus. It can identify lesions in terms of volume, proportion, and numbers. The accuracy obtained from the system is $92 \%$ on available datasets. China scientists developed a healthcare application named InferVISION to investigate COVID-19 patients. InferVISION utilized NVIDIA's Clara SDK [83]. It can identify the positive cases within a very small amount of time. Shenzhen-based company has developed a MicroMultiCopter that can carry medical samples from infected and dense areas. It can also be used for food and medical items delivery. LinkingMed technique to analyze the CT scan in less than sixty seconds. It provides $92 \%$ accuracy on test datasets.

Canadian based DarwinAI developed a neural network to analyze the X-rays for COVID-19 infection. Some hospitals do not have testing kits and radiologists to analyze the infection. In that case, an X-ray is an alternative to testing kits. DarwinAI developed a COVID-Net at University of Waterloo. DarwinAI trained from $17000 \mathrm{X}$-rays images. They are working on COVID-Net for identifying the risk of infection associated with workers [84].

Mumbai-based company Qure.ai developed an AI-based chest X-ray system named qXR [85]. The qXR is used to detect COVID patients from chest X-rays. qXR utilized deep learning models to detect lung abnormalities. It will help trainee doctors for their second opinion about the patient. 
479 The accuracy obtained to detect the COVID infected patients is approximately $95 \%$ over 11,000 480 patients. Lunit's software named "INSIGHT CXR" is used to scan the abnormalities of the lungs 481 [86]. These tools will help to handle the coronavirus pandemic.

482

483

484

485

486

487

488

489

490

491

492

493

494

495

496

497

498

499

500

501

502

503

504

505

506

507

508

509

510

511

512

513

514

515

516

517

518

Ron Li implemented an Epic model that can assess whether the patients have to shift to ICU or not [87]. He explored the "Deterioration Index" to identify whether the patient's condition is deteriorating or not. Epic trained 130,000 patients for assessing the validity of the Deterioration Index. He modified the model for the evaluation of COVID-19 patients in March. Six different organizations have been evaluated the performance of the Epic model on 3000 COVID patients and proved its performance.

Johns Hopkins University (JHU) developed a diagnostics tool for coronavirus infection [87]. Researchers in JHU developed a vigilant system for respiratory failure that can be caused by COVID. The respiratory diagnosis model will help the doctors to assess the infected patients. It will also envisage the need for ventilators and critical hospital instruments.

Maghdid et al. [88] proposed a mobile application to scan the CT images. CAD4COVID is an AIbased software to distinguish infected patients from chest X-rays [89]. Huazhong University of Science and Technology developed an algorithm for estimation of COVID-19 infected person with $80 \%$ accuracy. However, they tested on 53 patients of two different Chinese hospitals. Supplement Table 11 depicts the description of AI tools used for the treatment of COVID-19.

\section{ii. Deep Learning Architectures for Radiological Images}

Hemdan et al. [90] developed an automated diagnose framework called COVIDX-Net for the analysis of X-ray images. COVIDX-Net utilizes seven different deep learning models and tested over 50 X-ray images. The accuracy obtained from COVIDX-Net is $90 \%$. Wang et al. [91] developed a deep convolutional neural network (CNN) model (COVID-Net) for the identification of infection in chest X-ray images. COVID-Net model tested over 13,800 chest X-ray images and obtained $93.3 \%$ accuracy in recognizing normal, typical pneumonia, and COVID-19 cases. Ioannis et al. [92] evaluated CNN for the classification of COVID-19 cases. They used the transfer learning technique on 1427 X-ray images, achieving $98.75 \%$ and $93.48 \%$ accuracy for two and three class classification, respectively. Khan et al. [93] proposed a CoroNet architecture for detection of COVID-19 in chest X-ray of infected patients. CoroNet utilized the concept of Xception model. The classification performance of CoroNet was $99 \%$ and $89.6 \%$ for binary and three-class, respectively.

Ozturk et al. [94] developed a DarkCovidNet model for the detection of infected patients using chest X-ray images. DarkCovidNet model has seventeen convolutional layers and different filtering for each layer. The classification accuracies obtained from DarkCovidNet were 98.08\% 
519 and $87.02 \%$ for binary and multi-class, respectively. Sethy et al. [95] suggested deep learning 520 based methodology for detection of infected patients using chest X-ray images. The proposed 521 methodology used CNN models with support vector machine (SVM). The classification accuracy 522 of ResNet50 model with SVM classifier is $95.38 \%$. To overcome the shortcoming of hyper523 parameter tuning associated with transfer models, Kaur et al. [96] utilized the strength Pareto 524 evolutionary algorithm-II (SPEA-II) for chest X-ray images. They modified AlexNet to extract the 525 features from X-ray images and applied on classification process. The proposed approach 526 outperforms the competitive models in terms of performance measures. Jain et al. [97] developed 527 a deep learning model for diagnosis of chest X-ray images. IncpetionV3, Xception, and ResNeXt 528 are used in the development of proposed model. The classification accuracies obtained from this 529 530 model are $99 \%$ and $96 \%$ for training and testing, respectively.

Chowdhury et al. [98] designed an automatic coronavirus detection technique from X-ray images. This technique was evaluated on Chest X-ray dataset and attained the classification accuracy of 99.7\%. Islam et al. [99] combined convolutional neural network (CNN) and long short-term memory (LSTM) to diagnose coronavirus infection in the patients. The performance of this model was validated on $4575 \mathrm{X}$-ray images. The sensitivity and specificity of this model were $99.3 \%$ and $99.2 \%$, respectively. Nour et al. [100] developed a CNN model to extract discriminative features from chest X-ray. These features were applied on three well-known machine learning algorithms namely, k-nearest neighbor (KNN), SVM, and decision tree for classification. The sensitivity and specificity obtained from SVM-based classifier are $89.39 \%$ and $99.75 \%$, respectively. A number

541

542

543

544

545

546

547

548

549

550

551

552

553

554

555

556

557

558

of research articles were published in the field of diagnosis of COVID-19 using chest X-ray images [101-112].

Wang et al. [113] implemented deep learning approaches to extract specific features from CT scan images. These features are used to detect coronavirus infection using the transfer learning model. The developed model is tested on 1065 images of COVID-19. The accuracy obtained from their model is $89.5 \%$. Tan et al. [114] hybridized super resolution generative adversarial network (SRGAN) model and VGG16 to detect infected patients by chest CT. SRGAN was used to enhance the resolution of CT images. VGG16 was used to differentiate the infected and healthy region of CT. The developed model is validated over 275 COVID-19 and 195 normal CT images. The classification accuracy obtained from the model was $97.87 \%$. Xu et al. [115] used different CNN models to detect infected patients. CT images are processed to extract the interesting regions. 3D CNN model is used to segment the CT images. Thereafter, the segmented images are further classified into three different classes. The overall accuracy of deep learning models was $86.70 \%$.

Singh et al. [116] developed an automatic chest CT analysis system to classify the infected persons whether these are positive or not. The CNN hyper-parameters are tuned through multi-objective differential evolution (MODE). CNN with optimized parameters is used for COVID-19 patient classification. The proposed model outperforms the other competitive models by $1.927 \%$. Li et al.

Peer] Comput. Sci. reviewing PDF | (CS-2020:09:52767:1:2:NEW 27 Apr 2021) 
559 [117] developed a COVNet model for extracting 2D and 3D global features from chest $\mathrm{CT}$ for the

560

561

562

563

564

565

566

567

568

569

570

571

572

573

574

575

576

577

578

579

580

581

582

583

584

585

586

587

588

589

590

591

592

593

594

595

596

597

598

classification of COVID-19 patients. The extracted features are utilized to differentiate CoVID-19 infected, non-pneumonia, and community-acquired pneumonia (CAP). The classification accuracy obtained from COVNet was $96.00 \%$. Mei et al. [118] utilized machine learning techniques for the analysis of chest CT scans of COVID-19 patients. The clinical symptoms and laboratory testing were integrated with CT scans for analysis.

Hasan et al. [119] combined both deep learning technique and Q-deformed entropy for classification of COVID-19 using CT scan images. The features were extracted from CT scan images using Q-deformed entropy and CNN. The extracted features were applied on LSTM for distinguishing the COVID-19 and non COVID-19. The proposed approach attained the classification accuracy of $99.68 \%$. Wu et al. [120] developed a multi-view fusion model for identification of coronavirus infection in the CT scan images. This model was evaluated on the CT images of 495 patients. This model takes approximately ten minutes for analysis of CT images of infected patients. They used Youden index for fusion process. For testing phase, the sensitivity and specificity obtained from multi view model are $81.1 \%$ and $61.5 \%$, respectively.

Ko et al. [121] developed a FCONet model for COVID-19 classification using chest CT. FCONet utilized VGG16, ResNet50, InceptionV3, and Xception. The performance of FCONet was validated on 3993 chest CT images. The accuracy obtained from ResNet50 was 96.97\%. Researchers use deep learning architectures for analysis of CT scan images [122, 123].

The comparative analysis of deep learning techniques on radiological images such as chest X-ray and CT scan images are illustrated in Supplement Tables 12 and 13, respectively, while the usage of deep learning models is summarized in Fig. 6.

\section{b. Non-invasive techniques}

Several techniques do not need any specialized radiological equipment for diagnosis and treatment of COVID-19. Cho et al. [124] used a GRU neural network to determine the respiratory patterns of patients. The developed model is trained on footage obtained from Kinect depth cameras [125] and recognizes the COVID-19 patients.

Cascella et al. [126] suggested that COVID-19 patients have respiratory patterns, which are different from the common cold and flu. However, the abnormal respiratory patterns have no direct correlation with the diagnosis and treatment of COVID-19. The wearable devices and mobile applications can be utilized in the diagnosis and treatment of COVID-19. These devices and apps may utilize the body temperature, heart rate, cough samples, and breath rate.

\section{Drug Design}

Peer) Comput. Sci. reviewing PDF | (CS-2020:09:52767:1:2:NEW 27 Apr 2021) 
599 AI has the potential to discover, design, and repurpose the existing drugs to combat the COVID60019 as shown in Fig. 7. During this pandemic situation, several research laboratories are trying to 601 develop vaccines/drugs against COVID-19. They are using AI techniques to discover new vaccines 602 or repurposing existing drugs [127].

603

604

605

606

607

608

\section{a. Envisaging virus-host interactome}

The prediction of protein structure is necessary to develop new drugs. Senior et al. [128] developed an AlphaFold model to envisage the structures of proteins associated with SARS-CoV-2. ResNet is used to extract the features from amino acid sequences [129]. Heo and Feig [130] implemented

609 dilated ResNet to envisage the protein structure of SARS-CoV-2. They refined the AlphaFold's

610 predicted structures using molecular dynamics. Ge et al. [131] suggested an approach to construct

611 a knowledge graph that involving human proteins, viral proteins, and drugs. The knowledge graph is used to envisage possibly candidate drugs. Nguyen et al. [132] developed a SARS-based model using mathematical deep learning to determine possible inhibitors. 84 SARS coronavirus inhibitors are envisaged from ChEML and PDBind databases. Zhou et al. [133] developed network-based model to repurpose the drugs for SARS-CoV-2. Hu et al. [134] implemented a neural network to predict the affinities of SARS-CoV-2 proteins. They found ten possible drugs with their binding affinity scores among 4895 drugs.

618

619

Exscienta designed an AI-based drug molecule for coronavirus as reported in news [135]. It is the

620 first company who has designed the drug molecule for coronavirus. The traditional drug discovery

621 research took 4-5 years for developing new drugs. However, it will take one year to develop the

622 molecular structure. Insilco Medicine company used generative adversarial networks to determine the molecule structure. This structure is used to discover drugs. Insilco company screened 100 molecules for synthesis and testing. AI can be used to develop antibodies and vaccines for COVID19 [136]. It can be done in two ways namely, from scratch and drug repurposing. Google' DeepMind developed an AlphaGo algorithm to envisage the protein structure of the virus, which

627 can help develop new vaccines against coronavirus [137]. However, the experimentation has to be performed for validation of the designed protein.

\section{9}

630 b. Envisaging interaction among coronavirus and drugs

631

632

633

634

635

636

637

638
AI can be used to screen the exiting drug molecules and find their suitability against the coronavirus. South Korea and the USA used an AI-based algorithm to identify the 'Atazanavir' drug for repurposed to the treatment of COVID-19 [138]. Researchers of Benevolent AI identified 'Baricitinib' and 'Myelofibrosis' drugs for the treatment of COVID-19 [139]. Singaporean Firm Gero used a deep learning technique to recognize 'Afatinib' for the treatment of COVID-19. Zhang et al. [140] used fully connected ANN to envisage binding affinities from the PDBbind database. They explored the existing molecules for the treatment of SARS-CoV-2 [141]. Beck et al. [139] developed Molecule Transformer-Drug Target Interaction (MT-DTI) model to determine 
639 antivirals drugs that may be effective against coronavirus. BERT algorithm used to compute the 640 binding affinities of existing drugs. Hofmarcher et al. [142] used Long Short-Term Memory 641 (LSTM) model on SMILES data to screen 900 mln compounds from the ZINC dataset. 30,000 642 possible compounds are selected for treatment of SARS-CoV-2. These treatments may be available 643 in near future. The main reason behind this that medical trials, checks, and control are needed 644 before the approval of these drugs. After the identification and screening of drugs, a vaccine 645 formulation may take a minimum of 18 months [143].

646

647

\section{Social Implications}

648

649

650

Nowadays, the different myths regarding the incorrect data of infection, unsuitable drugs, and

651 misclassified infected zones have been propagated on social media. However, these rumors and hate speech can be greatly affected by the social life of human beings. WHO put these things in

652 infodemic, i.e., the huge amount of data is available with a mixture of accurate and inaccurate data.

653 Due to this, persons are unable to find verified sources whether the given information is correct or not in this pandemic situation.

655

656

657

\section{a. Analysis of Social Media}

658

659 In this epidemic situation, social media should assess the quality and accuracy of the information posted. Nowadays, Facebook and Google are working against the misinformation, phishing funding websites, and viruses floating on their platforms. When you search the COVID on

660

661 YouTube, then it links to the user either government organization or WHO for retrieving correct information. Videos posted on YouTube are screened and dropped immediately from the site after

662 the false information is confirmed. Eichstaedt et al. [144] analyzed the tweet posted on Twitter by

663

664 the user during this epidemic situation of COVID-19. AI-based text analyzer utilizing the number

665 of cases and death in a particular region to investigate the mental health of the user. MIT developed 666 a neural network-based model to determine the effectiveness of quarantine measures and the spread of virus infection [145]. Khataee et al. [146] reported the effects of social distancing during the COVID epidemic. They can assess the local elements of the pandemic. They reported that the 669 US has not taken precautionary measures to stop infection caused by a coronavirus. Rosenberg et al. [7] analyzed the tweets related to coronavirus-related information. They explored the various myths about the virus. Galotti et al. [147] explored the social media tweets posted on Twitter. They 671 developed an Inodemic Risk Index (IRI) to determine verified human beings, unverified human 672 beings, verified, and unverified bots. IRI utilizes the number of users and messages posted by users 673 and their reliability. They highlighted the impact of infodemics, social outcomes, and controlled 674 pandemics. Cinelli et al. [148] studied the contents of social media. They assessed the development 675 of the discourse on Twitter, YouTube, Instagram, and other social media. They analyzed the 676 comments, likes, and action upon comments for 45 days. Mejova et al. [149] studied the 677 advertisements related to coronavirus posted on Facebook. Facebook Ad Library is used to 678 examine all the advertisements that have the phrases "coronavirus" and "covid" across the world. 
679 They established that $5 \%$ of advertisements having misclassified and misconception information. 680 Zarocosta [150] studied the information shared and posted on different social media regarding 681 COVID-19. AI tools can be used to track the spreading of rumors regarding the coronavirus. 682 Pandey et al. [151] reported the breach in delivering genuine information to users in India. They 683 retrieved the information and found their verified sources using artificial intelligence and NLP. 684 They tested their approach on Sanitation and Hygienic information for this epidemic situation. AI685 based chatbots can be used to propagate COVID-19 information that can filter the misinformation. 686 WHO developed a multilingual chatbot to explore the information posted on social media and 687 news channels [152]. This virtual assistant can be used to verifying the information before further 688 processing.

689

690

691

\section{b. Analysis of Hate Speech}

Hate speech is a major concern in the last few months. The verbal and non-verbal abuse statements

692

693 may give rise to physical violence against the corona warriors. Such instances were reported in the lockdown situation of various countries. Velasquez et al. [153] analyzed the hate speeches

694

695

696

697

698

699

700

701

702

703

704

705

706

707

708

709

710

711

712

713

714

715

716

717

regarding COVID-19 posted on different social media and their movement from one media to another. They have also analyzed the methods for transmission and found that hate speeches are rapidly spread in the epidemic situation of COVID. Schild et al. [154] reported the Sinophopic behavior of tweets posted on Twitter and other media. They trained machine learning models on the information obtained from the contents of COVID-19. Web can be used for spreading misinformation and hate speech on COVID-19 information. AI-based tools play a vital role in fighting against hate speech [155].

Supplement Table 14 depicts the impact of rumors and hate speech on social life.

\section{Future Research Directions}

The possible research directions for application of AI on COVID-19 are described below:

\section{a. Interpretable prediction}

The results obtained from the AI-based prediction model should be interpretable and easy to use $[156,157]$. Therefore, soon one can utilize information extraction or image captioning kin of techniques to provide more interpretable prediction results.

\section{b. Mobile-based Al Tools}

The AI based models can be deployed on lightweight devices such as mobile. Our objective is not to achieve diagnostic tools for hospitals and clinics. On can work on AI-based COVID-19 prediction and diagnostic tools on lightweight devices [158]. 


\section{8 c. Drug Discovery}

719 Till now many researchers have worked on developing drugs for COVID-19 infected patients. 720 However, no effective drug is available for COVID-19 diagnosis. Therefore, one may utilize the

721

722

723

724

725

726

727

728

729

730

731

732

733

734

735

736

737

738

739

740

741

742

743

744

745

746

747

748

749

750

751

752

753

754

755

756 existing medicines to build an efficient drug for COVID-19 infected patients [159].

\section{d. Al-based Drones to combat COVID-19}

As we knew that the main source of infection transmission is human contact. Due to this, AI-based robots are used to disinfect the patient's rooms and interact with patients. For disinfection, robots are transmitting ultraviolet light over infected space to remove the virus. Robots are transferred the face image and voice of doctor on their screen during the interaction with patients. Hence, the medical staff is safe due to less contact with patients. Drones are widely used for transferring patient samples and medical apparatus. These are also used to disinfect the unreachable infected areas. During the lockdown, the drones are used to track the person who is come out from home. It provides faster delivery and less risk of infection. The drones supported by AI and computer vision techniques can play an essential role in the aerial monitoring of disease spread; for logistics and medical supply delivery [160], as well as for social distance checking [161]. They also were used to perform aerial spray and disinfection of residential areas [162]. However, there are data security, and privacy concerns that need to be resolved for successful application of AI-supported drone technology in public spaces on a large scale.

\section{e. 3D Printing techniques for developing COVID-19 prevention and fighting tools}

The 3D printing techniques may be used to design the face masks and face shields to be used for protection against COVID-19 [163]. Combined with face-scanning technology and computeraided design (CAD) tools these can be made to fit the individual face and head measurements. The 3D techniques also can be used to build other COVID-19 fighting tools such as main components of respiratory support equipment [164]. These components can be designed using low-cost consumer filament extrusion printers. The 3D printing technology can quickly address the deficiencies of medical materials and spare parts of medical equipment, however, the processing time, high cost, and lack of manpower can be potential barriers for applying 3D printing on a larger scale [165]. AI techniques can play a role in optimizing the 3D design process and reducing the cost of printing $[166,167,168]$.

\section{Findings, Lessons and Recommendations}

However, numerous challenges and research limitations have been indicated in the academic literature and need to be addressed in the future. Some of these challenges are related to nature and behaviour of COVID-19 because understanding how the virus spreads and how people can be 
757

758

759

760

761

762

763

764

765

766

767

768

769

770

771

772

773

774

775

776

777

778

779

780

781

782

783

784

785

786

787

788

789

790

791

792

793

794

795

infected caused by the complexity of this epidemic disease is extremely difficult. The lack of largescale datasets in the academic literature for COVID-19 is considered a challenging task for AI researchers because it hinders the understanding of viral patterns and features. In order to make AI and big data platforms and applications a trustful solution to fight the COVID-19 virus, a critical challenge is the collection of large-scale datasets and making them open for research.

AI and big data-based algorithms should be optimized further to enhance the accuracy and reliability of the data analytics for better COVID-19 diagnosis and treatment. AI is able to provide viable solutions for fighting the COVID-19 pandemic in several ways. For example, AI has proved very useful for supporting outbreak prediction, coronavirus detection as well as infodemiology and infoveillance by leveraging learning-based techniques such as ML and DL from COVID-19centric modeling, classification, and estimation. Moreover, AI has emerged as an attractive tool for facilitating vaccine and drug manufacturing. By using the datasets provided by healthcare organizations, governments, clinical labs and patients, AI leverages intelligent analytic tools for developing effective and safe vaccine/drug against COVID-19, which would be beneficial from both the economic and scientific perspectives. Moving forward, it is imperative for AI-designers and researchers to work together with medical professionals to create and develop these systems that are applicable to real-world datasets

\section{Conclusions}

In this paper, the impact of artificial intelligence (AI) techniques is assessed on early cautionary and vigilant systems that focus on COVID-19 warning and diagnostics. The COVID-19 datasets and visualization techniques are discussed with their applications. The AI-based diagnosis and treatment of COVID patients are assessed. The impact of AI on drug discovery and design is evaluated. The effect of COVID-19 is also evaluated on social and economic aspects. From the literature review, we can conclude that the AI techniques are widely used to identify the novel drug discovery beforehand of the outbreak of COVID-19. The AI techniques can help to search for the optimal drug against COVID-19. AI can be used to build the biomedical knowledge structure that connects the drugs and viruses to repurpose the existing drugs that are used to treat other diseases. The existing drug and coronavirus interaction can be modelled by AI techniques. Other promising applications of the AI methods are contact tracing, drone-based surveillance, and smart face mask design.

\section{References}

1. Wu, F., Zhao, S., Yu, B., Chen, Y.-M., Wang, W., Song, Z.-G., Hu, Y., Tao, Z.-W., Tian, J.-H., Pei, Y.-Y., Yuan, M.-L., Zhang, Y.-L., Dai, F.-H., Liu, Y., Wang, Q.-M., Zheng, J.-J., Xu, L., Holmes, E. C., \& Zhang, Y.-Z. (2020). A new coronavirus associated with human respiratory disease in China. Nature, 579(7798), 265-269. doi:10.1038/s41586-020-2008-3

Peer] Comput. Sci. reviewing PDF | (CS-2020:09:52767:1:2:NEW 27 Apr 2021) 
796 2. Huang, C., Wang, Y., Li, X., Ren, L., Zhao, J., Hu, Y., Zhang, L., Fan, G., Xu, J., Gu, X., Cheng, 797 Z., Yu, T., Xia, J., Wei, Y., Wu, W., Xie, X., Yin, W., Li, H., Liu, M., Cao, B. (2020). Clinical features of patients infected with 2019 novel coronavirus in Wuhan, China. The Lancet, 395(10223), 497-506. doi:10.1016/s0140-6736(20)30183-5

3. Zou, H., Shu, Y., \& Feng, T. (2020) How Shenzhen, China avoided widespread community transmission: a potential model for successful prevention and control of COVID-19, Infectious Diseases of Poverty, 9, 89. doi:10.1186/s40249-020-00714-2

4. Ayeningbara, I.O. (2020). COVID-19: An International Public Health Concern. Central Asian Journal of Global Health, 9(1), 1-11. DOI: 10.5195/cajgh.2020.466

5. Shi, H., Han, X., Jiang, N., Cao, Y., Alwalid, O., Gu, J., Fan, Y., \& Zheng, C. (2020). Radiological

806

807

808 findings from 81 patients with COVID-19 pneumonia in Wuhan, China: a descriptive study. The Lancet Infectious Diseases, 20(4), 425-434. doi:10.1016/s1473-3099(20)30086-4

6. Jiang, F., Deng, L., Zhang, L., Cai, Y., Cheung, C. W., \& Xia, Z. (2020). Review of the Clinical 809

810

811 Characteristics of Coronavirus Disease 2019 (COVID-19). Journal of general internal medicine, 35(5), 1545-1549. doi:10.1007/s11606-020-05762-w

812

813

814

7. Rosenberg, H., Syed, S., \& Rezaie, S. (2020) The twitter pandemic: The critical role of twitter in the dissemination of medical information and misinformation during the COVID-19 pandemic. CJEM. 22(4), 418-421.

8. Regmi, K, \& Lwin, C.M. (2020) Impact of non-pharmaceutical interventions for reducing

815

816

9. Verity, R., Okell, L. C., Dorigatti, I., Winskill, P., Whittaker, C., Imai, N., Cuomo-Dannenburg, G.,

817

818

819

820

821

822

823

824

825

826

827

828

829

830

831

832 Thompson, H., Walker, P. G. T., Fu, H., Dighe, A., Griffin, J. T., Baguelin, M., Bhatia, S., Boonyasiri, A., Cori, A., Cucunubá, Z., FitzJohn, R., Gaythorpe, K., ... Ferguson, N. M. (2020). Estimates of the severity of coronavirus disease 2019: a model-based analysis. The Lancet Infectious Diseases, 20(6), 669-677. doi:10.1016/s1473-3099(20)30243-7

10. Lalmuanawma, S., Hussain, J., \& Chhakchhuak, L. (2020) Applications of machine learning and artificial intelligence for Covid-19 (SARS-CoV-2) pandemic: A review, Chaos Solitons Fractals, 138, 110059.

11. Alimadadi, A., Aryal, S., Manandhar, I., Munroe, P.B., Joe, B. \& Cheng, X. (2020) Artificial intelligence and machine learning to fight COVID-19. Physiological Genomics, 52, 200-202.

12. Rao, G.G., Agarwal, A., \& Batura, D. (2020). Testing times in coronavirus disease (COVID-19): A tale of two nations. Med. J Armed Forces India, 76(3), 243-249

13. Allam, Z. (2020). The rise of machine intelligence in the COVID-19 pandemic and its impact on health policy. Surveying the COVID-19 Pandemic and its Implications, 89-96.

14. Liu K-C, Xu P, Lv W-F, Qiu X-H, Yao J-L, \& Jin-Feng G. (2020) CT manifestations of coronavirus disease-2019: a retrospective analysis of 73 cases by disease severity. Eur J Radiol 108941

15. Bullock J., Luccioni A., Pham K.H., Lam C.S.N., \& Luengo-Oroz M. (2020) Mapping the landscape of artificial intelligence applications against COVID-19. Journal of Artificial Intelligence Research, 69, 807-845

834

835

836

16. T. Singhal (2020) A review of coronavirus disease-2019 (COVID-19), Indian J. Pediatr. 87, 281-

837

838

839 286

17. L.T. Phan, T.V. Nguyen, Q.C. Luong, T.V. Nguyen, H.T. Nguyen, H.Q. Le, Q. D. Pham, Importation and human-to-human transmission of a novel coronavirus in Vietnam, N. Engl. J. Med. 382 (9) (2020) 872-874. 
840 18. Majumder MS, \& Mandl KD. Early in the epidemic: impact of preprints on global discourse about

841

842

843

844

845

846

847

848

849

850

851

852

853

854

855

856

857

858

859

860

861

862

863

864

865

866

867

868

869

870

871

872

873

874

875

876

877

878

879

880

881

882

COVID-19 transmissibility. The Lancet. 8(5), p. E627-E30, 2020

19. Senthilraja M. (2021) Application of artificial intelligence to address issues related to the COVID19 Virus, SLAS Technol. 26(2), 123-126

20. Chen Q, Allot A, \& Lu Z. (2020) Keep up with the latest coronavirus research. Nature. 579(7798):193.

21. Albahri, A. S., Hamid, R. A., Alwan, J. k., Al-qays, Z. T., Zaidan, A. A., Zaidan, B. B., Albahri, A. O. S., AlAmoodi, A. H., Khlaf, J. M., Almahdi, E. M., Thabet, E., Hadi, S. M., Mohammed, K. I., Alsalem, M. A., Al-Obaidi, J. R., \& Madhloom, H. T. (2020). Role of biological Data Mining and Machine Learning Techniques in Detecting and Diagnosing the Novel Coronavirus (COVID19): A Systematic Review. Journal of Medical Systems, 44(7). doi:10.1007/s10916-020-01582-x

22. Lalmuanawma, S., Hussain, J., \& Chhakchhuak, L. (2020). Applications of machine learning and artificial intelligence for covid-19 (SARS-CoV-2) pandemic: A review. Chaos, Solitons and Fractals, 139 doi:10.1016/j.chaos.2020.110059

23. Ozsahin, I., Sekeroglu, B., Musa, M. S., Mustapha, M. T., \& Uzun Ozsahin, D. (2020). Review on diagnosis of COVID-19 from chest CT images using artificial intelligence. Computational and Mathematical Methods in Medicine, doi:10.1155/2020/9756518.

24. Pham, Q. -.T, Nguyen, D. C., Huynh-The, T., Hwang, W., \& Pathirana, P. N. (2020). Artificial intelligence (AI) and big data for coronavirus (COVID-19) pandemic: A survey on the state-of-thearts. IEEE Access, 8, 130820-130839. doi:10.1109/ACCESS.2020.3009328

25. Rasheed, J., Jamil, A., Hameed, A. A., Aftab, U., Aftab, J., Shah, S. A., \& Draheim, D. (2020). A survey on artificial intelligence approaches in supporting frontline workers and decision makers for the COVID-19 pandemic. Chaos, Solitons and Fractals, 141 doi:10.1016/j.chaos.2020.110337

26. Tseng, V. S., Jia-Ching Ying, J., Wong, S. T. C., Cook, D. J., \& Liu, J. (2020). Computational intelligence techniques for combating COVID-19: A survey. IEEE Computational Intelligence Magazine, 15(4), 10-22. doi:10.1109/MCI.2020.3019873

27. Tayarani, M.-H. (2021) Applications of artificial intelligence in battling against covid-19: a literature review. Chaos Solitons Fractals, 142: 110338

28. Kumar, V., Kaur, M. \& Singh, D. (2021) Impact of Metaheuristic Techniques in Pandemic of COVID-19. Coronaviruses. Doi: 10.2174/2666796701999201218142021

29. X. Xie, Z. Zhong, W. Zhao, C. Zheng, F. Wang, J. Liu, Chest CT for typical 2019- nCoV pneumonia: relationship to negative RT-PCR testing, Radiology (2020)

30. Chen Y, Liu Q, Guo D. Coronaviruses: genome structure, replication, and pathogenesis. J Med Virol. 2020; 92:418-423.

31. Su, S., Wong, G., Shi, W., Liu, J., Lai, A. C. K., Zhou, J., Liu, W., Bi, Y., \& Gao, G. F. (2016). Epidemiology, Genetic Recombination, and Pathogenesis of Coronaviruses. Trends in Microbiology, 24(6), 490-502. doi:10.1016/j.tim.2016.03.003

32. Abd-Alrazaq, A., Alajlani, M., Alhuwail, D., Schneider, J., Al-Kuwari, S., Shah, Z., Hamdi, M., \& Househ, M. (2020). Artificial Intelligence in the Fight Against COVID-19: Scoping Review. Journal of Medical Internet Research, 22(12), e20756. doi:10.2196/20756

33. Naude W. Artificial Intelligence against COVID-19: An Early Review, 2020. IZA Institute of Labor Economics. DP No.13110

34. https://bluedot.global

PeerJ Comput. Sci. reviewing PDF | (CS-2020:09:52767:1:2:NEW 27 Apr 2021) 
883 35. Bogoch, I., A. Watts, Thomas-Bachli, A., Huber, C., Kraemer, M., and Khan, K. (2020).

884

885

886

887

888

889

890

891

892

893

894

895

896

897

898

899

900

901

902

903

904

905

906

907

908

909

910

911

912

913

914

915

916

917

918

919

920

921

922

923

924

925

Pneumonia of Unknown Aaetiology in Wuhan, China: Potential for International Spread via Commercial Air Travel. Journal of Travel Medicine, 27(2):1-3.

36. Yankoski M, Weninger T, Scheirer W. (2020) An AI early warning system to monitor online disinformation, stop violence, and protect elections. Bulletin of the Atomic Scientists. 26(2), pp. $85-90$.

37. Ming, L. C., Untong, N., Aliudin, N. A., Osili, N., Kifli, N., Tan, C. S., Goh, K. W., Ng, P. W., AlWorafi, Y. M., Lee, K. S., \& Goh, H. P. (2020). Mobile Health Apps on COVID-19 Launched in the Early Days of the Pandemic: Content Analysis and Review. JMIR MHealth and UHealth, 8(9), e19796. doi:10.2196/19796

38. Comba, J.L.D. (2020). Data visualization for the understanding of COVID-19. Computing in Science \& Engineering, 22, 81-86.

39. Anastassopoulou C., Russo L., Tsakris A., Siettos C. (2020) Data-based analysis, modelling and forecasting of the COVID-19 outbreak. PLoS ONE, 15(3): e0230405.

40. Ahamed S, Samad MD. (2020) Information Mining For COVID-19 Research From A Large Volume Of Scientific Literature. arXiv preprint arXiv:200402085. 2020

41. Abd-Alrazaq A., Schnider J., Mifsud B., Alam T., Househ M., Hamdi M., \& Shah Z. (2021) A Comprehensive Overview of the COVID-19 Literature: Machine Learning-Based Bibliometric Analysis. Journal of Medical Internet Research, 23(3): e23703

42. Lwin M.O., Lu J., Sheldenkar A., Schulz P.J., Shin W., Gupta R., Yang Y. (2020) Global sentiments surrounding the covid-19 pandemic on twitter: analysis of twitter trends. JMIR Publ Health Surveill 6(2):e19447

43. Lamsal, R. (2020) Design and analysis of a large-scale COVID-19 tweets dataset. Applied Intelligence. DoI: 10.1007/s10489-020-02029-z

44. Kleinberg B, van der Vegt I, \& Mozes M. Measuring Emotions in the COVID-19 Real World Worry Dataset. arXiv preprint arXiv:200404225. 2020

45. Minaee S., Kafieh R., Sonka M., Yazdani S., \& Soufi G.J. (2020) Deep-COVID: Predicting COVID-19 from chest X-ray images using deep transfer learning. Medical Image Analysis, 65:101794

46. Miller, J.A., Khatib F., Hammond H., Cooper S., \& Horowitz S. (2020) Introducing Foldit education mode. Nature Structural \& Molecular Biology, 27, 769-770

47. Xu, B., Gutierrez, B., Mekaru, S., Sewalk, K., Goodwin, L., Loskill, A., Cohn, E. L., Hswen, Y., Hill, S. C., Cobo, M. M., Zarebski, A. E., Li, S., Wu, C.-H., Hulland, E., Morgan, J. D., Wang, L., O’Brien, K., Scarpino, S. V., Brownstein, J. S., ... Kraemer, M. U. G. (2020). Epidemiological data from the COVID-19 outbreak, real-time case information. Scientific Data, 7(1). doi:10.1038/s41597-020-0448-0

48. Shuja J., Alanazi E., Alasmary W., \& Alashaikh A. (2021) COVID-19 open source datasets: a comprehensive survey. Applied Intelligence, 51, 1296-1325

49. Yan, L., Zhang, H.-T., Xiao, Y., Wang, M., C. Sun, Liang, J., Li, S., M. Zhang, Guo, Y., Xiao, Y., Tang, X., Cao, H., Tan, X., Huang, N., A. Luo, B. J., Cao, Z., H. Xu, Yuan, Y. (2020). Prediction of Criticality in Patients with Severe Covid-19 Infection Using Three Clinical Features: A Machine Learning-Based Prognostic Model with Clinical Data in Wuhan. medRxiv, doi: 10.1101/2020.02.27.20028027 
926

927

928

929

930

931

932

933

934

935

936

937

938

939

940

941

942

943

944

945

946

947

948

949

950

951

952

953

954

955

956

957

958

959

960

961

962

963

964

965

966

967

968

50. Jiang, X., Coffee, M., Bari, A., Wang, J., Jiang, X., Huang, J., Shi, J., Dai, J., Cai, J., Zhang, T., Wu, Z., He, G., and Huang, Y. (2020). Towards an Artificial Intelligence Framework for DataDriven Prediction of Coronavirus Clinical Severity. Computers, Materials and Continua, 63(1), 537-551.

51. Alotaibi A., Shiblee M., \& Alshahrani A. (2021) Prediction of Severity of COVID-19-Infected Patients Using Machine Learning Techniques, Computers, 10, 31

52. Chatterjee K., Chatterjee K., Kumar A., \& Shankar S. (2020) Healthcare impact of COVID-19 epidemic in India: a stochastic mathematical model. Med J Armed Forces India. 76(2), 147-155.

53. Ghosal S., Sengupta S., Majumder M., \& Sinha B. (2020) Linear Regression Analysis to predict the number of deaths in India due to SARS-CoV-2 at 6 weeks from day 0 (100 cases - March14th 2020). Diabetes Metab Syndr. 14, 311-315.

54. Imran, A., Posokhova, I., Qureshi, H. N., Masood, U., Riaz, M. S., Ali, K., John, C. N., Hussain, M. I., \& Nabeel, M. (2020). AI4COVID-19: AI enabled preliminary diagnosis for COVID-19 from cough samples via an app. Informatics in Medicine Unlocked, 20, 100378. doi:10.1016/j.imu.2020.100378

55. Feng, C., Wang, L., Chen, X., Zhai, Y., Zhu, F., Chen, H., Wang, Y., Su, X., Huang, S., Tian, L., Zhu, W., Sun, W., Zhang, L., Han, Q., Zhang, J., Pan, F., Chen, L., Zhu, Z., Xiao, H., ... Li, T. (2021). A novel artificial intelligence-assisted triage tool to aid in the diagnosis of suspected COVID-19 pneumonia cases in fever clinics. Annals of Translational Medicine, 9(3), 201-201. doi:10.21037/atm-20-3073

56. Rao A.S.S., \& Vazquez J. A. (2020) Identification of COVID-19 Can be Quicker through Artificial Intelligence framework using a Mobile Phone-Based Survey in the Populations when Cities/Towns Are Under Quarantine. Infect Control Hospl Epidemiol. 41(7), 826-830

57. Tang, Z., Zhao, W., Xie, X., Zhong, Z., Shi, F., Ma, T., Liu, J., \& Shen, D. (2021). Severity assessment of COVID-19 using CT image features and laboratory indices. Physics in Medicine \& Biology, 66(3), 035015. doi:10.1088/1361-6560/abbf9e

58. Yue, H., Yu, Q., Liu, C., Huang, Y., Jiang, Z., Shao, C., Zhang, H., Ma, B., Wang, Y., Xie, G., Zhang, H., Li, X., Kang, N., Meng, X., Huang, S., Xu, D., Lei, J., Huang, H., Yang, J., ... Qi, X. (2020). Machine learning-based CT radiomics method for predicting hospital stay in patients with pneumonia associated with SARS-CoV-2 infection: a multicenter study. Annals of Translational Medicine, 8(14), 859-859. doi:10.21037/atm-20-3026

59. Patrikar S., Poojary D., Basannar D.R., Faujdar D.S., \& Kunte R. (2020) Projections for novel coronavirus (COVID-19) and evaluation of epidemic response strategies for India. Med J Armed Forces India, 76(3), 268-275

60. Ting D., Carin L., Dzau V., \& Wong T. Y. (2020). Digital technology and COVID-19. Nature medicine, 26(4), 459-461.

61. Martin TW, Lin L. (2020) Fever-Detecting Goggles and Disinfectant Drones: Countries Turn to Tech to Fight Coronavirus. The Wall Street Journal. 3 May 2020

62. Xavier J. (2020) A deeper look at Apple and Google's joint technology to combat COVID-19. The Hindu. 16 April 2020

63. Robitzski D. (2020) This Smart Ring Uses AI to Spot COVID-19 - Before Symptoms Begin. Studies. 11 April 2020. https://futurism.com/neoscope/smart-ring-ai-spot-covid19-beforesymptoms-begin. 
969 64. Bruggeman L. (2020) Researchers investigate whether wearable apps could unveil hidden

970

971

972

973

974

975

976

977

978

979

980

981

982

983

984

985

986

987

988

989

990

991

992

993

994

995

996

997

998

999

1000

1001

1002

1003

1004

1005

1006

1007

1008

1009

1010

1011 coronavirus cases. Coronavirus Health \& Science. 2 April, 2020

65. Russey C. (2020) Proxxi Launches Halo Wearable Wristband to Maintain Social Distance at Work During Covid-19 Pandemic. Health \& Wellness. 23 April 2020

66. Obeidat S. (2020) How Artificial Intelligence Is Helping Fight The COVID-19 Pandemic. Business Connect. 30 March 2020

67. Schreurs E. (2020) How we Created an Open-Source COVID-19 Chatbot. Towards Data Science. 2020.

68. Maddox T. (2020) Google Cloud launches new AI chatbot for COVID-19 information. TechRepublic. 8 April 2020

69. Hutchinson A (2020). WhatsApp Launches World Health Organization Chatbot to Answer COVID-19 Queries. Social Media Today. 21 March 2020.

70. Chanthadavong A (2020) South Australia Health introduces bot to answer COVID-19 queries. ZDNet. Coronavirus: Business and Technology in a Pandemic. 28 April 2020

71. Long, C., Xu, H., Shen, Q., Zhang, X., Fan, B., Wang, C., Zeng, B., Li, Z., Li, X., \& Li, H. (2020). Diagnosis of the Coronavirus disease (COVID-19): rRT-PCR or CT? European Journal of Radiology, 126, 108961. doi:10.1016/j.ejrad.2020.108961

72. Z.Y. Zu, M.D. Jiang, P.P. Xu, W. Chen, Q.Q. Ni, G.M. Lu, \& L.J. Zhang (2020) Coronavirus disease 2019 (COVID-19): a perspective from China, Radiology, 296(2). https://doi. org/10.1148/radiol.2020200490.

73. J.P. Kanne, B.P. Little, J.H. Chung, B.M. Elicker, L.H. Ketai (2020) Essentials for radiologists on COVID-19: an update — radiology scientific expert panel, Radiology, 296(2)

74. E.Y. Lee, M.Y. Ng, \& P.L. Khong (2020) COVID-19 pneumonia: what has CT taught us? Lancet Infect. Dis. 20 (4), 384-385.

75. Wynants, L., Van Calster, B., Collins, G. S., Riley, R. D., Heinze, G., Schuit, E., Bonten, M. M. J., Dahly, D. L., Damen, J. A., Debray, T. P. A., de Jong, V. M. T., De Vos, M., Dhiman, P., Haller, M. C., Harhay, M. O., Henckaerts, L., Heus, P., Kammer, M., Kreuzberger, N., ... van Smeden, M. (2020). Prediction models for diagnosis and prognosis of covid-19: systematic review and critical appraisal. BMJ, m1328. doi:10.1136/bmj.m1328

76. Bernheim, A., Mei, X., Huang, M., Yang, Y., Fayad, Z. A., Zhang, N., Diao, K., Lin, B., Zhu, X., Li, K., Li, S., Shan, H., Jacobi, A., \& Chung, M. (2020). Chest CT Findings in Coronavirus Disease19 (COVID-19): Relationship to Duration of Infection. Radiology, 295(3), 200463. doi:10.1148/radiol.2020200463

77. Pan, F., Ye, T., Sun, P., Gui, S., Liang, B., Li, L., Zheng, D., Wang, J., Hesketh, R. L., Yang, L., $\&$ Zheng, C. (2020). Time Course of Lung Changes at Chest CT during Recovery from Coronavirus Disease 2019 (COVID-19). Radiology, 295(3), 715-721. doi:10.1148/radiol.2020200370

78. Ai, T., Yang, Z., Hou, H., Zhan, C., Chen, C., Lv, W., Tao, Q., Sun, Z., \& Xia, L. (2020). Correlation of Chest CT and RT-PCR Testing for Coronavirus Disease 2019 (COVID-19) in China: A Report of 1014 Cases. Radiology, 296(2), E32-E40. doi:10.1148/radiol.2020200642

79. Ng, M.-Y., Lee, E. Y. P., Yang, J., Yang, F., Li, X., Wang, H., Lui, M. M., Lo, C. S.-Y., Leung, B., Khong, P.-L., Hui, C. K.-M., Yuen, K., \& Kuo, M. D. (2020). Imaging Profile of the COVID19 Infection: Radiologic Findings and Literature Review. Radiology: Cardiothoracic Imaging, 2(1), e200034. doi:10.1148/ryct.2020200034 
1012 80. Fang, Y., Zhang, H., Xie, J., Lin, M., Ying, L., Pang, P., \& Ji, W. (2020). Sensitivity of Chest CT

1013 for COVID-19: Comparison to RT-PCR. Radiology, 296(2), E115-E117.

1014

1015 doi:10.1148/radiol.2020200432

81. Weinstock MB, RJea Echenique A. Chest x-ray findings in 636 ambulatory patients with COVID19 presenting to an urgent care center: a normal chest $\mathrm{x}$-ray is no guarantee. The Journal of Urgent Care Medicine. 2020; p. 13-18.

1017

1018

82. McFarland A (2020) Baidu's AI Technology Being Used to Combat Coronavirus. UNITE.AI:

1019

1020 COVID-19. 13 March 2020

83. Chen K (2020) COVID-19 and artificial intelligence: protecting health-care workers and curbing

1021

1022

1023

1024

1025

1026

1027

1028

1029

1030 the spread. Digital Health. 2: e166-e167. doi: 10.1016/S2589-7500(20)30054-6

84. Borkowski A.A., Viswanadhan N.A., Thomas L.B., Guzman R.D., Deland L.A., \& Mastorides S.M. (2020) Using Artificial Intelligence for COVID-19 Chest X-ray Diagnosis. Fed Pract. 37(9), 398-404

85. Bora G. (2020) Qure.ai can detect Covid-19 lung infections in less than a minute, help triage patients. Economic Times. 30 April 2020

86. Lunit. www.lunit.io/covid19

87. Strickland E. (2020) AI Can Help Hospitals Triage COVID-19 Patients. IEEE Spectrum. 17 April 2020

88. Maghdid HS, Ghafoor KZ, Sadiq AS, Curran K, Rabie K. A Novel AI-enabled Framework to

1031

1032 Diagnose Coronavirus COVID-19 using Smartphone Embedded Sensors: Design Study. 2020.

89. Delft Imaging (2020). https://www.delft.care/cad4covid/

1033

90. E.E.D. Hemdan, M.A. Shouman, \& M.E. Karar (2020) COVIDX-Net: A Framework of Deep

1034

1035 Learning Classifiers to Diagnose COVID-19 in X-Ray Images, arXiv preprint arXiv:2003.11055.

91. L. Wang, Z.Q. Lin, \& A. Wong (2020) COVID-Net: A tailored deep convolutional neural network design for detection of COVID-19 cases from Chest X-ray Images, Scientific Reports, 10:19549

1037

92. I. D. Apostolopoulos, T. Bessiana, COVID-19: automatic detection from X-Ray images utilizing transfer learning with convolutional neural networks, Physical and Engineering sciences in Medicine, 43, 635-649

93. Khan A.I., Shah J.L., \& Bhat M.M. (2020) CoroNet: A deep neural network for detection and diagnosis of COVID-19 from chest x-ray images, Comput Methods Programs Biomed, 196:105581.

94. Ozturk T., Talo M., Yildirim E.A., Baloglu U.B., Yildirim O., Acharya U.R. (2020) Automated detection of COVID-19 cases using deep neural networks with X-ray images. Comput. Biol. Med. 121:103792

95. Sethy P.K., Behera S.K., Ratha P.K., \& Biswas P. (2020) Detection of coronavirus disease (COVID-19) based on deep features and support vector machine. International Journal of Mathematical, Engineering and Management Sciences, 5(4), 643-651

96. Kaur, M., Kumar, V., Yadav, V., Singh, D., Kumar, N., \& Das, N.N. (2021) Metaheuristic-based Deep COVID-19 Screening Model from Chest X-Ray Images. Journal of Healthcare Engineering, 8829829, doi: 10.1155/2021/8829829

97. Jain R., Gupta M., Taneja S., \& Hemanth D.J. (2021) Deep learning based detection and analysis of COVID-19on chest X-ray images, Applied Intelligence, 15, 1690-1700

1053

1054

98. Chowdhury, M. E. H., Rahman, T., Khandakar, A., Mazhar, R., Kadir, M. A., Mahbub, Z. B., Islam, 1055 K. R., Khan, M. S., Iqbal, A., Emadi, N. A., Reaz, M. B. I., \& Islam, M. T. (2020). Can AI Help in 
1056

1057

1058

1059

1060

1061

1062

1063

1064

1065

1066

1067

1068

1069

1070

1071

1072

1073

1074

1075

1076

1077

1078

1079

1080

1081

1082

1083

1084

1085

1086

1087

1088

1089

1090

1091

1092

1093

1094

1095

1096

1097

1098

1099

Screening Viral and COVID-19 Pneumonia? IEEE Access, 8, 132665-132676. doi:10.1109/access.2020.3010287

99. Islam MdZ, Islam MdM, \& Asraf A (2020) A combined deep CNN-LSTM network for the detection of novel coronavirus (COVID-19) using X-ray images. Inform Med Unlocked. 20:100412

100. Nour M., Cömert Z., \& Polat K (2020) A novel medical diagnosis model for COVID-19 infection detection based on deep features and Bayesian optimization. Appl Soft Comput, 97: 106580

101. Oh Y., Park S., \& Ye J.C. (2020) Deep learning COVID-19 features on CXR using limited training data sets. IEEE Trans Med Imaging, 39(8), 2688-2700

102. Pereira R.M., Bertolini D., Teixeira L.O., Silla C.N., \& Costa Y.M.G. (2020) COVID-19 identification in chest X-ray images on flat and hierarchical classification scenarios. Comput Methods Programs Biomed, 194, 105532

103. Elaziz M.A., Hosny K.M., Salah A., Darwish M.M., Lu S., \& Sahlol A.T. (2020) New machine learning method for image-based diagnosis of COVID-19. PLoS One. 15(6):e0235187.

104. Jain G., Mittal D., Thakur D., \& Mittal M.K. (2020) A deep learning approach to detect Covid-19 coronavirus with X-ray images. Biocybern Biomed Eng. 40(4), 1391-1405

105. Panwar H., Gupta P.K., Siddiqui M.K., Morales-Menendez R., \& Singh V. (2020) Application of deep learning for fast detection of COVID-19 in X-rays using nCOVnet. Chaos Solitons Fract, 109944

106. Tsiknakis, N., Trivizakis, E., Vassalou, E., Papadakis, G., Spandidos, D., Tsatsakis, A., Sánchez-García, J., López-González, R., Papanikolaou, N., Karantanas, A., \& Marias, K. (2020). Interpretable artificial intelligence framework for COVID-19 screening on chest X-rays. Experimental and Therapeutic Medicine, 20(2), 727-735. doi:10.3892/etm.2020.8797

107. Gianchandani N., Jaiswal A., Singh D., Kumar V. \& Kaur M. (2020) Rapid COVID-19 diagnosis using ensemble deep transfer learning models from chest radiographic images. Journal of Ambient Intelligence and Humanized Computing.

108. Singh D., Kumar V., Yadav V., \& Kaur M. (2021) Deep Neural Network-Based Screening Model for COVID-19-Infected Patients Using Chest X-Ray Images. International Journal of Pattern Recognition and Artificial Intelligence, 35(3), 2151004.

109. Das N.N., Kumar N., Kaur M., Kumar V., \& Singh D. (2020) Automated Deep Transfer LearningBased Approach for Detection of COVID-19 Infection in Chest X-rays. IRBM.

110. Altan A., \& Karasu S. (2020) Recognition of COVID-19 disease from X-ray images by hybrid model consisting of $2 \mathrm{D}$ curvelet transform, chaotic salp swarm algorithm and deep learning technique. Chaos, Solitons \& Fractals, 140:110071

111. Lopez-Cabrera J.D., Orozco-Morales R., Portal-Diaz J.A., Lovelle-Enriquez O., \& Perez-Diaz M. (2021) Current limitations to identify COVID-19 using artificial intelligence with chest X-ray imaging. Health and Technology, 11, 411-424

112. Ghaderzadeh M., \& Asadi F. (2021) Deep learning in the detection and diagnosis of COVID-19 using radiology modalities: a systematic review. Journal of Healthcare Engineering, 6677314.

113. S. Wang, B. Kang, J. Ma, X. Zeng, M. Xiao, J. Guo, \& B. Xu (2021) A deep learning algorithm using CT images to screen for Corona Virus Disease (COVID-19), Eur Radiol. 24, 1-9

114. Tan W., Liu P., Li X., Liu Y., Zhou Q., Chen C., Gong Z., Yin X., \& Zhang Y. (2020) Classification of COVID-19 pneumonia from chest CT images based on reconstructed super-resolution images and VGG neural network, Health Information Science and Systems, 9: 10.

Peer] Comput. Sci. reviewing PDF | (CS-2020:09:52767:1:2:NEW 27 Apr 2021) 
1100 115. Xu, X., Jiang, X., Ma, C., Du, P., Li, X., Lv, S., Yu, L., Ni, Q., Chen, Y., Su, J., Lang, G., Li, Y.,

1101

1102

1103

1104

1105

1106

1107

1108

1109

1110

1111

1112

1113

1114

1115

1116

1117

1118

1119

1120

1121

1122

1123

1124

1125

1126

1127

1128

1129

1130

1131

1132

1133

1134

1135

1136

1137

1138

1139

1140

1141

1142
Zhao, H., Liu, J., Xu, K., Ruan, L., Sheng, J., Qiu, Y., Wu, W., ... Li, L. (2020). A Deep Learning System to Screen Novel Coronavirus Disease 2019 Pneumonia. Engineering, 6(10), 1122-1129. doi:10.1016/j.eng.2020.04.010

116. Singh, D., Kumar, V., Vaishali, \& Kaur, M. (2020). Classification of COVID-19 patients from chest CT images using multi-objective differential evolution-based convolutional neural networks. European journal of clinical microbiology \& infectious diseases : official publication of the European Society of Clinical Microbiology, 1-11.

117. Li, L., Qin, L., Xu, Z., Yin, Y., Wang, X., Kong, B., Bai, J., Lu, Y., Fang, Z., Song, Q., Cao, K., Liu, D., Wang, G., Xu, Q., Fang, X., Zhang, S., Xia, J., \& Xia, J. (2020). Using Artificial Intelligence to Detect COVID-19 and Community-acquired Pneumonia Based on Pulmonary CT: Evaluation of the Diagnostic Accuracy. Radiology, 296(2), E65-E71. doi:10.1148/radiol.2020200905

118. Mei, X., Lee, H.-C., Diao, K., Huang, M., Lin, B., Liu, C., Xie, Z., Ma, Y., Robson, P. M., Chung, M., Bernheim, A., Mani, V., Calcagno, C., Li, K., Li, S., Shan, H., Lv, J., Zhao, T., Xia, J., ... Yang, Y. (2020). Artificial intelligence-enabled rapid diagnosis of patients with COVID-19. Nature Medicine, 26(8), 1224-1228. doi:10.1038/s41591-020-0931-3

119. A. M. Hasan, M. M. Al-Jawad, H. A. Jalab, H. Shaiba, R. W. Ibrahim, and A. R. Al-Shamasneh, (2020) Classification of COVID-19 coronavirus, pneumonia and healthy lungs in CT scans using Q-deformed entropy and deep learning features. Entropy, 22(5)

120. Wu, X., Hui, H., Niu, M., Li, L., Wang, L., He, B., Yang, X., Li, L., Li, H., Tian, J., \& Zha, Y. (2020). Deep learning-based multi-view fusion model for screening 2019 novel coronavirus pneumonia: A multicentre study. European Journal of Radiology, 128, 109041. doi:10.1016/j.ejrad.2020.109041

121. Ko, H., Chung, H., Kang, W. S., Kim, K. W., Shin, Y., Kang, S. J., Lee, J. H., Kim, Y. J., Kim, N. Y., Jung, H., \& Lee, J. (2020). COVID-19 Pneumonia Diagnosis Using a Simple 2D Deep Learning Framework With a Single Chest CT Image: Model Development and Validation. Journal of medical Internet research, 22(6), e19569. doi:10.2196/19569

122. Jaiswal, A., Gianchandani, N., Singh, D., Kumar, V. \& Kaur, M. (2020) Classification of the COVID-19 infected patients using DenseNet201 based deep transfer learning. Journal of Biomolecular Structure and Dynamics. Doi: 10.1080/07391102.2020.1788642

123. Singh, D., Kumar, V., \& Kaur, M. (2021) Densely connected convolutional networks-based COVID-19 screening model. Applied Intelligence. Doi: 10.1007/s10489-020-02149-6

124. Cho, K., van Merrienboer, B., Gulcehre, C., Bahdanau, D., Bougares, F., Schwenk, H., \& Bengio, Y. (2014). Learning Phrase Representations using RNN Encoder-Decoder for Statistical Machine Translation. Proceedings of the 2014 Conference on Empirical Methods in Natural Language Processing (EMNLP). Proceedings of the 2014 Conference on Empirical Methods in Natural Language Processing (EMNLP). doi:10.3115/v1/d14-1179

125. Wang Y, Hu M, Li Q, Zhang XP, Zhai G, Yao N. Abnormal respiratory patterns classifier may contribute to large-scale screening of people infected with COVID-19 in an accurate and unobtrusive manner. arXiv preprint arXiv:200205534.2020

126. Cascella M, Rajnik M, Cuomo A, Dulebohn SC, Di Napoli R. Features, Evaluation, and Treatment Coronavirus (COVID-19). In: Stat Pearls [Internet]. StatPearls Publishing; 2020. 
1143 127. Kaushik A.C. \& Raj U. (2020). AI-driven drug discovery: A boon against COVID-19? AI Open,

1144

1145

1146

1147

1148

1149

1150

1151

1152

1153

1154

1155

1156

1157

1158

1159

1160

1161

1162

1163

1164

1165

1166

1167

1168

1169

1170

1171

1172

1173

1174

1175

1176

1177

1178

1179

1180

1181

1182

1183

1184

1185

1186 1-4.

128. Senior, A. W., Evans, R., Jumper, J., Kirkpatrick, J., Sifre, L., Green, T., Qin, C., Žídek, A., Nelson, A. W. R., Bridgland, A., Penedones, H., Petersen, S., Simonyan, K., Crossan, S., Kohli, P., Jones, D. T., Silver, D., Kavukcuoglu, K., \& Hassabis, D. (2020). Improved protein structure prediction using potentials from deep learning. Nature, 577(7792), 706-710. doi:10.1038/s41586-019-19237

129. Jumper J, Tunyasuvunakool K, Kohli P, Hassabis D, AlphaFold Team. Computational predictions of protein structures associated with COVID-19; 2020.

130. Heo L, Feig M. (2020) Modeling of Severe Acute Respiratory Syndrome Coronavirus2 (SARSCoV-2) Proteins by Machine Learning and Physics-Based Refinement. bioRxiv doi:10.1101/2020.03.25.008904

131. Ge, Y., Tian, T., Huang, S., Wan, F., Li, J., Li, S., Yang, H., Hong, L., Wu, N., Yuan, E., Cheng, L., Lei, Y., Shu, H., Feng, X., Jiang, Z., Chi, Y., Guo, X., Cui, L., Xiao, L., ... Zeng, J. (2020). A data-driven drug repositioning framework discovered a potential therapeutic agent targeting COVID-19. Cold Spring Harbor Laboratory. doi:10.1101/2020.03.11.986836

132. Nguyen D, Gao K, Chen J, Wang R, \& Wei G. (2020) Potentially highly potent drugs for2019nCoV. bioRxiv, doi: 10.1101/2020.02.05.936013

133. Zhou Y., Hou Y., Shen J., Huang Y., Martin W., \& Cheng F. (2020) Network-based drug repurposing for novel coronavirus 2019-nCoV/SARS-CoV-2, Cell Discovery, 2:14

134. Hu F, Jiang J, \& Yin P. (2020) Prediction of Potential Commercially Inhibitors against SARSCoV-2 by Multi-Task Deep Model. arXiv preprint arXiv:200300728.

135. Kirk D. (2020) UK-US Initiative to Screen Drugs Using AI for Coronavirus Treatments. LABIOTECH.eu. 3 April 2020

136. Vanderslott, S., Pollard, A., and Thomas, T. (2020). Coronavirus Vaccine: Here are the Steps it will Need to Go Through During Development. The Conversation, 30 March 2020.

137. Silver, D., Hubert, T., Schrittwieser, J., I.Antonoglou, Lai, M., Guez, A., Lanctot, M., Sifre, L., Kumaran, D., Graepel, T., Lillicrap, T., Simonyan, K., and Hassabis, D. (2018). A General Reinforcement Learning Algorithm that Masters Chess, Shogi, and Go Through Self-Play. Science, 362(6419):1140-1144.

138. Beck B.R., Shin B., Choi Y., Park S., \& Kang K. (2020). Predicting Commercially Available Antiviral Drugs that may act on the Novel Coronavirus (2019-nCoV), Wuhan, China through a Drug-Target Interaction Deep Learning Model. Computational and Structural Biotechnology Journal, 18, 784-790

139. Stebbing, J., Phelan, A., I.Griffin, C.Tucker, Oechsle, O., Smith, D., \& Richardson, P. (2020). COVID-19: Combining Antiviral and Anti-Inflammatory Treatments. The Lancet, 20:400-401.

140. Zhang, H., Saravanan, K. M., Yang, Y., Hossain, M. T., Li, J., Ren, X., Pan, Y., \& Wei, Y. (2020). Deep Learning Based Drug Screening for Novel Coronavirus 2019-nCov. Interdisciplinary sciences, computational life sciences, 12(3), 368-376. doi:10.1007/s12539-020-00376-6

141. Zhang, H., Yang, Y., Li, J., Wang, M., Saravanan, K. M., Wei, J., Tze-Yang Ng, J., Tofazzal Hossain, Md., Liu, M., Zhang, H., Ren, X., Pan, Y., Peng, Y., Shi, Y., Wan, X., Liu, Y., \& Wei, Y. (2020). A novel virtual screening procedure identifies Pralatrexate as inhibitor of SARS-CoV-2 RdRp and it reduces viral replication in vitro. PLOS Computational Biology, 16(12), e1008489. doi:10.1371/journal.pcbi.1008489 
1187

1188

1189

1190

1191

1192

1193

1194

1195

1196

1197

1198

1199

1200

1201

1202

1203

1204

1205

1206

1207

1208

1209

1210

1211

1212

1213

1214

1215

1216

1217

1218

1219

1220

1221

1222

1223

1224

1225

1226

1227

1228

1229

142. Hofmarcher, M., Mayr, A., Rumetshofer, E., Ruch, P., Renz, P., Schimunek, J., Seidl, P., Vall, A., Widrich, M., Hochreiter, S., \& Klambauer, G. (2020). Large-Scale Ligand-Based Virtual Screening for SARS-CoV-2 Inhibitors Using Deep Neural Networks. SSRN Electronic Journal. doi:10.2139/ssrn.3561442

143. Levin, J. M., Oprea, T. I., Davidovich, S., Clozel, T., Overington, J. P., Vanhaelen, Q., Cantor, C. R., Bischof, E., \& Zhavoronkov, A. (2020). Artificial intelligence, drug repurposing and peer review. Nature Biotechnology, 38(10), 1127-1131. doi:10.1038/s41587-020-0686-x

144. Eichstaedt, J. C., Schwartz, H. A., Kern, M. L., Park, G., Labarthe, D. R., Merchant, R. M., Jha, S., Agrawal, M., Dziurzynski, L. A., Sap, M., Weeg, C., Larson, E. E., Ungar, L. H., \& Seligman, M. E. (2015). Psychological language on Twitter predicts county-level heart disease mortality. Psychological science, 26(2), 159-169. doi: 10.1177/0956797614557867

145. Dandekar, R., Rackauckas, C., \& Barbastathis, G. (2020). A machine learning-aided global diagnostic and comparative tool to assess effect of quarantine control in COVID-19 spread. Patterns, 1(9):100145

146. Khataee H., Scheuring I., Czirok A., \& Neufeld Z. (2020) effects of social distancing on the spreading of COVID-19 inferred from mobile phone data. Scientific Reports, 11:1661

147. Gallotti R, Valle F, Castaldo N, Sacco P, \& Domenico MD. (2020) Assessing the risks of "infodemics" in response to COVID-19 epidemics. Nature Human Behaviour, 4, 1285-1293

148. Cinelli, M., Quattrociocchi, W., Galeazzi, A., Valensise, C. M., Brugnoli, E., Schmidt, A. L., Zola, P., Zollo, F., \& Scala, A. (2020). The COVID-19 social media infodemic. Scientific Reports, 10(1). doi:10.1038/s41598-020-73510-5

149. Mejova Y, \& Kalimeri K. (2020) Advertisers Jump on Coronavirus Bandwagon: Politics, News, and Business. arXiv preprint arXiv:200300923.

150. Zarocostas J. How to fight an infodemic. The Lancet. 2020; 395(10225):676.

151. Pandey R, Gautam V, Bhagat K, Sethi T. A Machine Learning Application for Raising WASH Awareness in the Times of COVID-19 Pandemic. arXiv preprintarXiv:200307074. 2020.

152. Miner, A.S., Laranjo, L., \& Kocaballi, A.B. (2020) Chatbots in the fight against the COVID-19 pandemic. npj Digital Medicine, 3.

153. N. Velásquez, R. Leahy, N. Johnson Restrepo, Y. Lupu, R. Sear, N. Gabriel, O. Jha, B. Goldberg, N.F. Johnson (2020) Hate multiverse spreads malicious COVID-19 content online beyond individual platform control. arXiv:2004.00673

154. Schild L, Ling C, Blackburn J, Stringhini G, Zhang Y, Zannettou S. "Go eat a bat, Chang!": An Early Look on the Emergence of Sinophobic Behavior on Web Communities in the Face of COVID-19. arXiv preprint arXiv:200404046. 2020

155. Atehortua N.A., \& Patino S. (2020) COVID-19, a tale of two pandemics: novel coronavirus and fake news messaging. Health Promot Int., daaa140

156. Roberts, M., Driggs, D., Thorpe, M., Gilbey, J., Yeung, M., Ursprung, S., Aviles-Rivero, A. I., Etmann, C., McCague, C., Beer, L., Weir-McCall, J. R., Teng, Z., Gkrania-Klotsas, E., Rudd, J. H. F., Sala, E., \& Schönlieb, C.-B. (2021). Common pitfalls and recommendations for using machine learning to detect and prognosticate for COVID-19 using chest radiographs and CT scans. Nature Machine Intelligence, 3(3), 199-217. doi:10.1038/s42256-021-00307-0

157. Wieczorek, M., Siłka, J., \& Woźniak, M. (2020). Neural network powered COVID-19 spread forecasting model. Chaos, Solitons \& Fractals, 140, 110203. doi:10.1016/j.chaos.2020.110203 
1230

1231

1232

1233

1234

1235

1236

1237

1238

1239

1240

1241

1242

1243

1244

1245

1246

1247

1248

1249

1250

1251

1252

1253

1254

1255

1256

1257

1258

1259
158. Iyengar, K., Upadhyaya, G. K., Vaishya, R., \& Jain, V. (2020). COVID-19 and applications of smartphone technology in the current pandemic. Diabetes \& Metabolic Syndrome: Clinical Research \& Reviews, 14(5), 733-737. doi: 10.1016/j.dsx.2020.05.033

159. Ho, D. (2020). Addressing COVID-19 Drug Development with Artificial Intelligence. Advanced Intelligent Systems, 2(5), 2000070 (1:4). doi: 10.1002/aisy.202000070

160. Angurala, M., Bala, M., Bamber, S.S., Kaur, R., \& Singh, P. (2020) An internet of things assisted drone based approach to reduce rapid spread of COVID-19, Journal of Safety Science and Resilience, 1(1), 31-35. doi:10.1016/j.jnlssr.2020.06.011

161. Kumar, A., Sharma, K., Singh, H., Naugriya, S. G., Gill, S. S., \& Buyya, R. (2021). A drone-based networked system and methods for combating coronavirus disease (COVID-19) pandemic. Future generations computer systems: FGCS, 115, 1-19. doi:10.1016/j.future.2020.08.046

162. Yaacoub, J. P., Noura, H., Salman, O., \& Chehab, A. (2020). Security analysis of drones systems: Attacks, limitations, and recommendations. Internet of Things, 11, 100218. doi:10.1016/j.iot.2020.100218

163. Swennen GRJ, Pottel L, Haers PE. Custom-made 3D-printed face masks in case of pandemic crisis situations with a lack of commercially available FFP2/3 masks. Int J Oral Maxillofac Surg. 2020;49(5):673-677. doi:10.1016/j.ijom.2020.03.015

164. Tino, R., Moore, R., Antoline, S., Ravi, P., Wake, N., Ionita, C. N., Morris, J. M., Decker, S. J., Sheikh, A., Rybicki, F. J., \& Chepelev, L. L. (2020). COVID-19 and the role of 3D printing in medicine. 3D Printing in Medicine, 6(1). doi: 10.1186/s41205-020-00064-7

165. Ishack, S., \& Lipner, S. R. (2020). Applications of 3D Printing Technology to Address COVID19-Related Supply Shortages. The American Journal of Medicine, 7:771-773. doi: 10.1016/j.amjmed.2020.04.002

166. Wang, Y., Zheng, P., Peng, T., Yang, H., \& Zou, J. (2020). Smart additive manufacturing: Current artificial intelligence-enabled methods and future perspectives. Science China Technological Sciences, 63:1600-1611.

167. Longhitano, G.A., Nunes, G.B., \& Candido, G. (2021) The role of 3D printing during COVID-19 pandemic: a review. Progress in Additive Manufacturing, 6:19-37.

168. Almalki, M., \& Azeez, F. (2020) Health Chatbots for Fighting COVID-19: a Scoping Review, Acta Inform Med., 28(4), 241-247. 
Figure 1

Number of preprints available related to COVID-19 on different platforms (From Jan, 2020 to April, 2021)

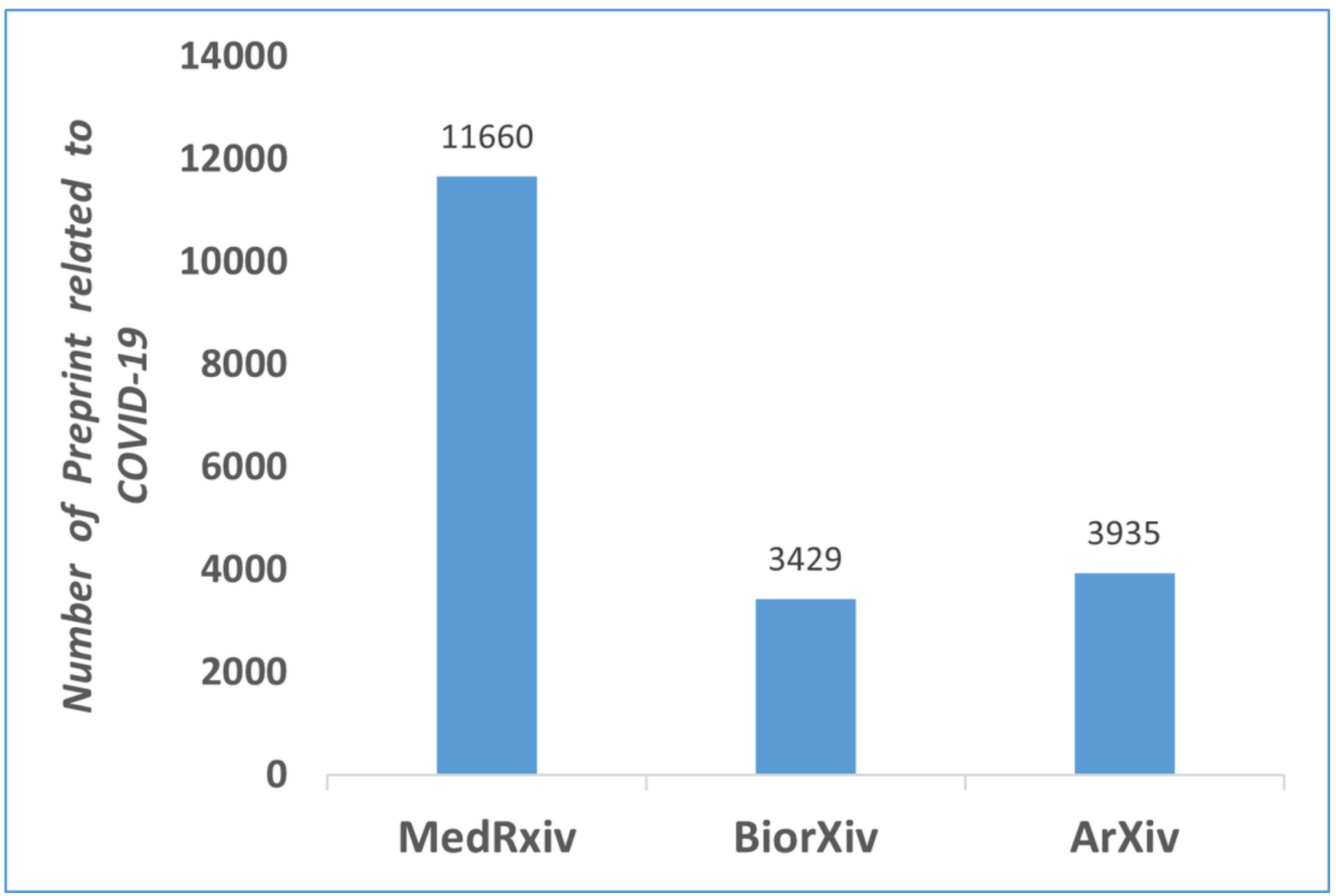


Figure 2

Classification of data gathering systems

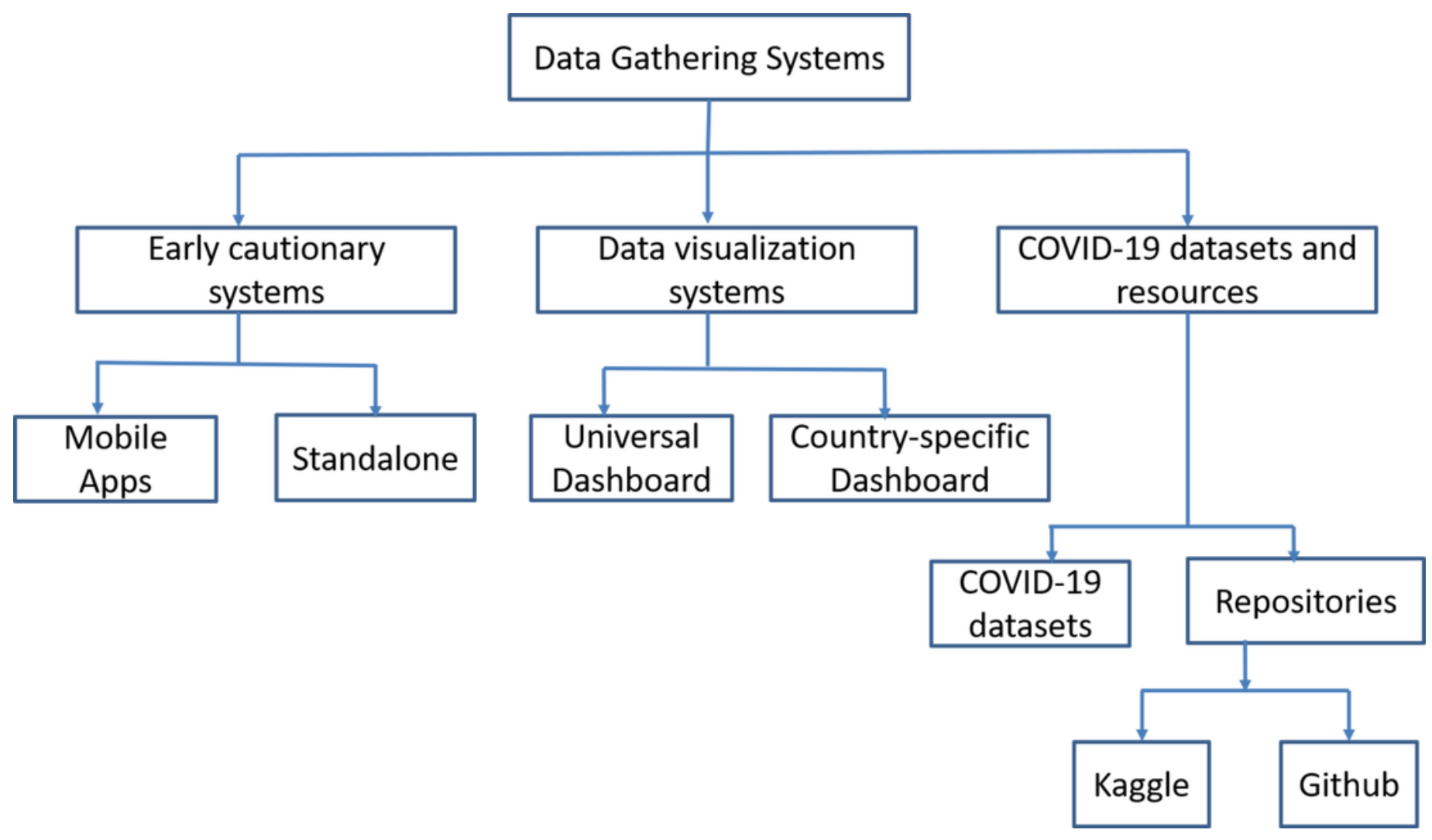


Figure 3

Classification of COVID-19 Datasets

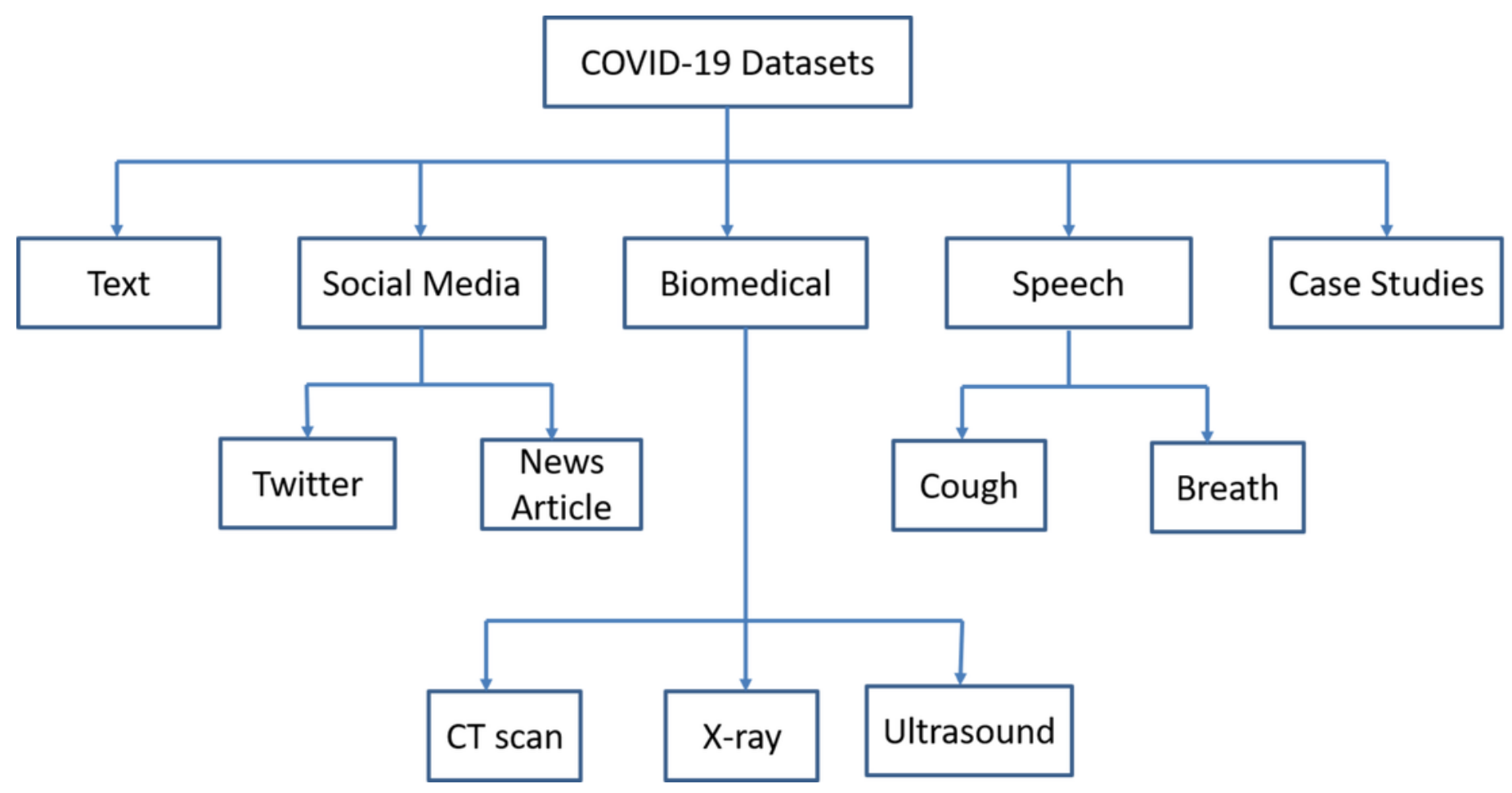


Figure 4

Screening techniques for COVID-19 symptoms

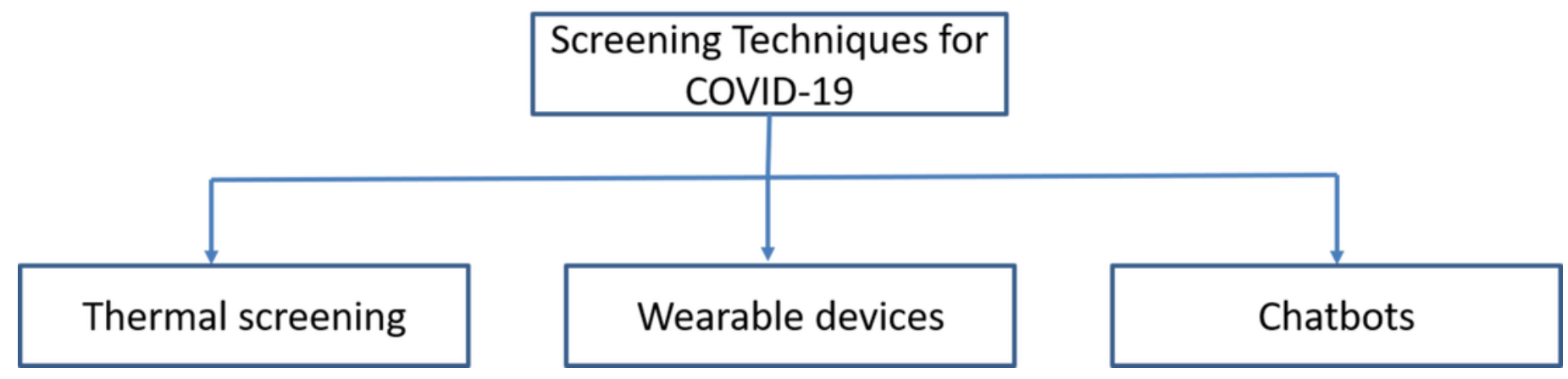




\section{Figure 5}

Different radiological modality used in deep learning techniques

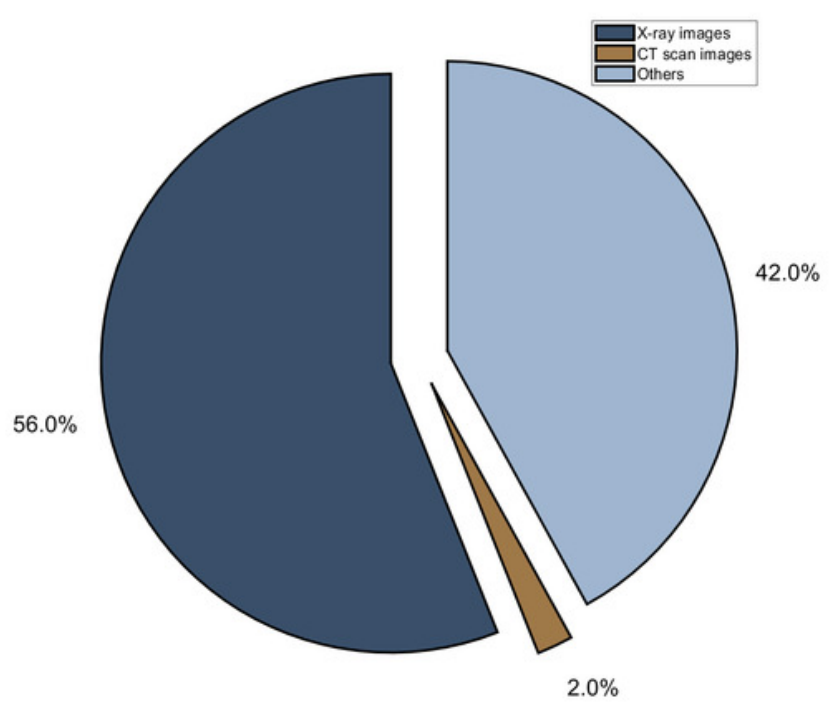


Figure 6

Different deep learning architectures used for diagnosis of COVID-19

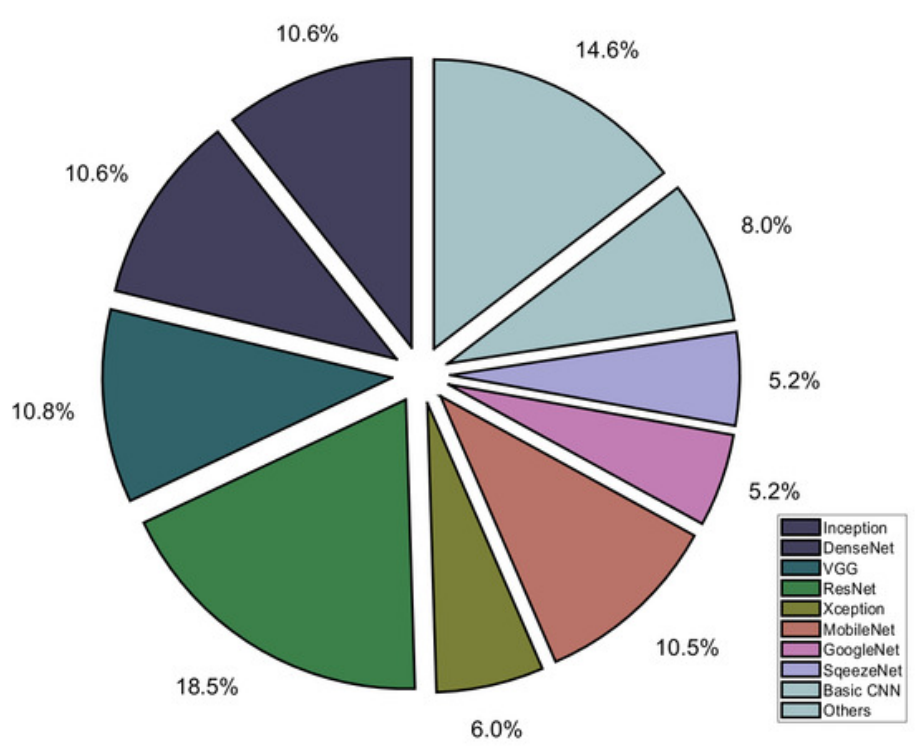


Figure 7

Deep learning and machine learning based drug synergy prediction for COVID-19

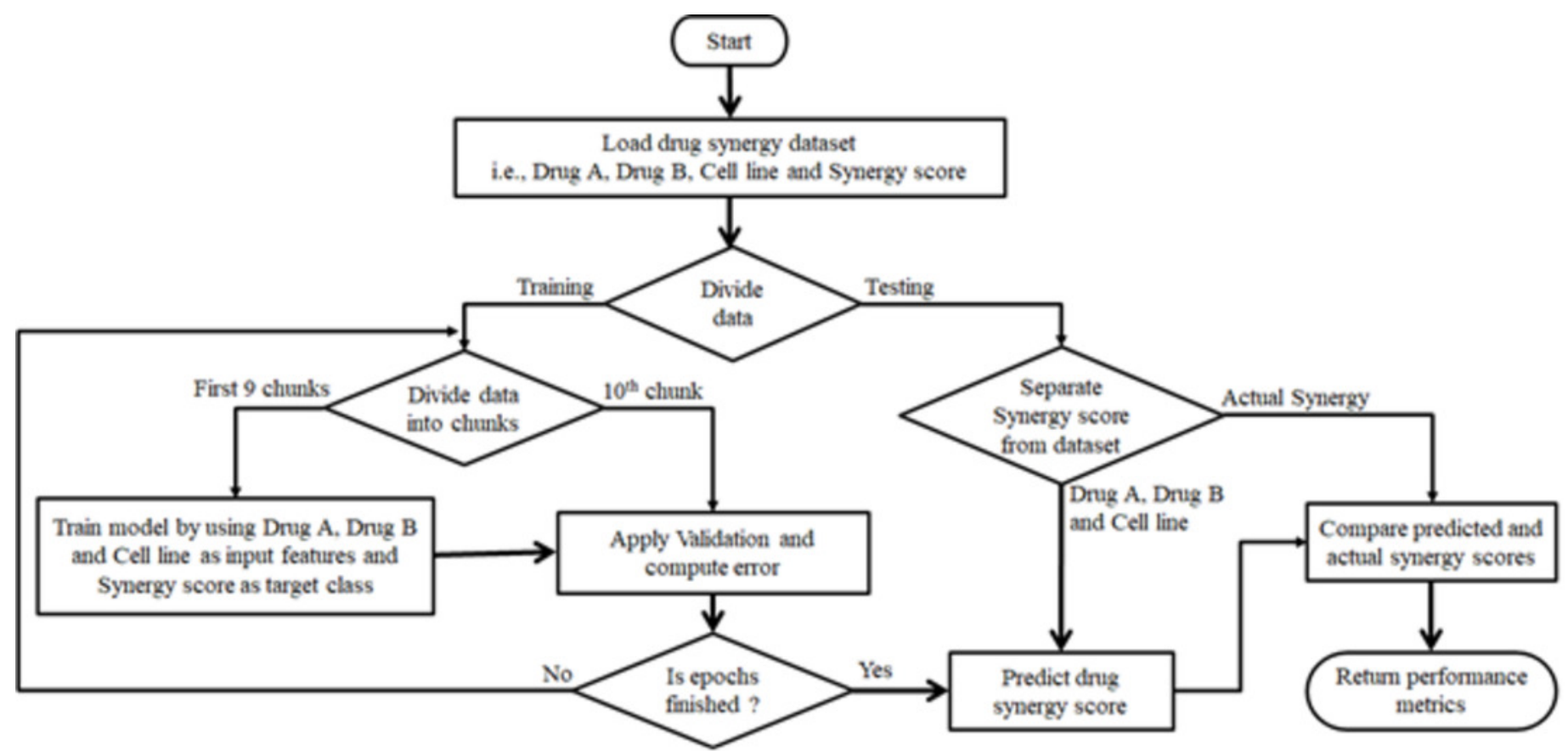

